\title{
Derived intersections and the Hodge theorem
}

\author{
Dima Arinkin, Andrei Căldăraru and Márton Hablicsek
}

\begin{abstract}
The algebraic Hodge theorem was proved in a beautiful 1987 paper by Deligne and Illusie, using positive characteristic methods. We argue that the central algebraic object of their proof can be understood geometrically as a line bundle on a derived scheme. In this interpretation, the Deligne-Illusie result can be seen as a proof that this line bundle is trivial under certain assumptions. We give a criterion for the triviality of this line bundle in a more general context. The proof uses techniques from derived algebraic geometry, specifically arguments which show the formality of certain derived intersections. Applying our criterion, we recover Deligne and Illusie's original result. We also apply these techniques to the result of Barannikov-Kontsevich, Sabbah, and Ogus-Vologodsky concerning the formality of the twisted de Rham complex.
\end{abstract}

\section{Introduction}

In their 1987 paper [DI87], Deligne and Illusie proved the following algebraic version of the Hodge theorem.

THEOREM 1.1. Let $X$ be a smooth proper scheme over a perfect field $\mathbf{k}$ of positive characteristic $p>\operatorname{dim} X$. Assume that $X$ lifts to the ring $W_{2}(\mathbf{k})$ of second Witt vectors of $k$. Then the Hodge-de Rham spectral sequence for $X$ degenerates at ${ }^{1} E$.

The corresponding result in characteristic zero (the Hodge theorem) follows easily from this result using a standard argument of Frobenius.

Deligne and Illusie deduce Theorem 1.1 from the following statement, whose proof forms the central part of their paper. Consider the Frobenius twist $X^{\prime}$ of $X$ and the corresponding relative Frobenius morphism $F: X \rightarrow X^{\prime}$. If we denote by $\Omega_{X}^{\circ}$ the algebraic de Rham complex of $X$, then the complex $F_{*} \Omega_{X}^{\circ}$ is an object of $\mathbf{D}\left(X^{\prime}\right)$, the derived category of coherent sheaves on $X^{\prime}$. Deligne and Illusie proved that the two statements below are equivalent.

(i) The scheme $X$ lifts to $W_{2}(\mathbf{k})$.

(ii) The complex $F_{*} \Omega_{X}^{\bullet}$ is formal in $\mathbf{D}\left(X^{\prime}\right)$.

(A complex is said to be formal if it is isomorphic to the direct sum of its cohomology sheaves.)

Received 21 December 2013, accepted in final form 9 August 2016.

2010 Mathematics Subject Classification 14F40, 14F22, 14F30.

Keywords: Hodge theorem, twisted de Rham complex, formality.

This journal is (C) Foundation Compositio Mathematica 2017. This article is distributed with Open Access under the terms of the Creative Commons Attribution Non-Commercial License, which permits non-commercial reuse, distribution, and reproduction in any medium, provided that the original work is properly cited. For commercial re-use, please contact the Foundation Compositio Mathematica.

The authors are supported by the National Science Foundation under Grants No. DMS-0901224, DMS-1101558, and DMS-1200721. 


\section{Derived INTERSECTIONS AND THE Hodge THEOREM}

Independently of the work of Deligne and Illusie, the first two authors proved the following result in $[\mathrm{AC} 12]$.

Theorem 1.2. Let $i: X \hookrightarrow S$ be a closed embedding of smooth schemes over a field $\mathbf{k}$ of characteristic $p>\operatorname{codim}_{S} X$, and assume that the normal bundle $N=N_{X / S}$ is the restriction of a vector bundle $\bar{N}$ on the first infinitesimal neighborhood of $X$ in $S$. Then the two statements below are equivalent.

(i) The trivial vector bundle $\mathscr{O}_{X}$ lifts to the first infinitesimal neighborhood of $X$ in $S$.

(ii) The structure complex $i^{*} i_{*} \mathscr{O}_{X}$ of the derived self-intersection of $X$ inside $S$ is formal.

(All functors are implicitly assumed to be derived.)

Remark 1.3. As stated, part (i) is vacuous. We will see that in order to understand the result of Deligne and Illusie, we need to work with a generalization of Theorem 1.2 to Azumaya spaces (see below), where statement (i) becomes a real condition.

Mustaţă noted that there is a strong similarity between Theorem 1.2 and the Deligne-Illusie equivalence. He asked if it is possible to recast the problem of Deligne-Illusie as a derived intersection problem such that their result would follow from Theorem 1.2. The goal of this paper is to give a positive answer to this question, by constructing a closed embedding $h: X^{\prime} \hookrightarrow \bar{S}$ for which $F_{*} \Omega_{X}^{*}$ is dual to the structure sheaf $h^{*} h_{*} \mathscr{O}_{X^{\prime}}$ of the derived self-intersection of $X^{\prime}$ inside $\bar{S}$. In particular, $F_{*} \Omega_{X}^{*}$ will be formal if and only if $h^{*} h_{*} \mathscr{O}_{X^{\prime}}$ is.

One feature of this construction is that $\bar{S}$ is not an ordinary scheme but an extended object called an Azumaya space; see Section 3 or [Căl00]. Such a space is a pair $(S, \mathscr{A})$ where $S$ is an ordinary scheme and $\mathscr{A}$ is a sheaf of Azumaya algebras on $S$. Coherent sheaves on the Azumaya space $(S, \mathscr{A})$ are coherent sheaves on $S$ together with a left $\mathscr{A}$-module structure. Morphisms in the category of Azumaya spaces (or, more precisely, 1-morphisms in this 2-category) are given by Morita equivalences.

Because of how morphisms are defined in the category of Azumaya spaces, it is not a priori true that the trivial bundle $\mathscr{O}_{X^{\prime}}$ extends to the first infinitesimal neighborhood for the embed$\operatorname{ding} h$. In fact, we will prove that in the Deligne-Illusie problem, this happens if and only if $X$ lifts to $W_{2}(\mathbf{k})$. Applying a version of Theorem 1.2 suited to Azumaya spaces (Corollary 4.9) in the setting we have engineered above recovers Theorem 1.1.

We now describe the construction of the embedding $h$. For this we need some classic facts about rings of crystalline differential operators (for more details, see Section 2). Denote the total space of the cotangent bundle of $X^{\prime}$ by $T^{*} X^{\prime}$, and consider the embedding $i: X^{\prime} \hookrightarrow T^{*} X^{\prime}$ of $X^{\prime}$ into $T^{*} X^{\prime}$ as the zero section. The sheaf of crystalline differential operators $D_{X}$ of $X$ has center $\mathscr{O}_{T^{*} X^{\prime}}$ and thus can be regarded as a sheaf of algebras $D$ over $T^{*} X^{\prime}$. Moreover, $D$ is an Azumaya algebra over $T^{*} X^{\prime}$, and along the zero section of $T^{*} X^{\prime}$, the Azumaya algebra $D$ splits,

$$
\left.D\right|_{X^{\prime}} \cong \operatorname{End}_{X^{\prime}}\left(F_{*} \mathscr{O}_{X}\right) \text {. }
$$

Thus $\mathscr{O}_{X^{\prime}}$ and $\left.D\right|_{X^{\prime}}$ are Morita equivalent, and we obtain an equivalence of abelian categories

$$
\mathfrak{C o h}\left(X^{\prime}\right) \cong \mathfrak{C o h}\left(X^{\prime},\left.D\right|_{X^{\prime}}\right),
$$

where the latter is the abelian category of coherent sheaves on $X^{\prime}$ endowed with the structure of left $\left.D\right|_{X^{\prime}}$-module.

From the point of view of Azumaya spaces, the above equivalence of categories of coherent 


\section{Arinkin, A. CăLdăraru And M. Hablicsek}

sheaves is simply an isomorphism

$$
m:\left(X^{\prime}, \mathscr{O}_{X^{\prime}}\right) \stackrel{\sim}{\rightarrow}\left(X^{\prime},\left.D\right|_{X^{\prime}}\right) .
$$

Moreover, the embedding $i: X^{\prime} \rightarrow T^{*} X^{\prime}$ gives rise to an embedding of Azumaya spaces

$$
i_{D}:\left(X^{\prime},\left.D\right|_{X^{\prime}}\right) \rightarrow\left(T^{*} X^{\prime}, D\right) .
$$

The first result of this paper is the following.

Theorem 1.4. Let $X$ be a smooth scheme over a perfect field $\mathbf{k}$ of characteristic $p>0$. Consider the composite morphism

$$
h:\left(X^{\prime}, \mathcal{O}_{X^{\prime}}\right) \stackrel{m}{\rightarrow}\left(X^{\prime},\left.D\right|_{X^{\prime}}\right) \stackrel{i_{D}}{\longrightarrow}\left(T^{*} X^{\prime}, D\right) .
$$

Then there are natural isomorphisms in $\mathbf{D}\left(X^{\prime}\right)$

$$
F_{*} \Omega_{X}^{\cdot} \cong h^{!} h_{*} \mathcal{O}_{X^{\prime}} \cong\left(h^{*} h_{*} \mathscr{O}_{X^{\prime}}\right)^{\vee} .
$$

Thus $F_{*} \Omega_{X}^{*}$ is formal if and only if $h^{*} h_{*} \mathscr{O}_{X^{\prime}}$ is.

Remark 1.5 Twisted derived intersections). This theorem shows that it is important to understand formality properties for derived intersections inside Azumaya spaces. This is a more general problem which we discuss now.

Let $\bar{S}=(S, \mathscr{A})$ be an Azumaya space, and let $X$ and $Y$ be subvarieties of $S$ such that $\mathscr{A}$ splits along $X$ and $Y$. By choosing splitting modules, one can regard the ordinary (untwisted) spaces $X$ and $Y$ as subvarieties of $(S, \mathscr{A})$. We want to compare the differential-graded (dg) spaces $W$ and $\bar{W}$ which are obtained as the derived intersections of $X$ and $Y$ inside $S$ and $\bar{S}$, respectively.

The central geometric observation of this paper is that there is a natural line bundle $\mathcal{L}$ on $W$ which measures the difference between the spaces $W$ and $\bar{W}$. (In a sense, this line bundle measures the extent to which the chosen splittings fail to agree along $W$; see Section 4.) If the dg-space $W$ is formal, the formality of $\bar{W}$ often reduces to the problem of checking whether $\mathcal{L}$ is trivial.

This discussion allows us to recast the original Deligne-Illusie problem in geometric language. For this problem, we need to study the derived self-intersection of the zero section $X^{\prime}$ inside the Azumaya space $\left(T^{*} X^{\prime}, D\right)$. The untwisted intersection $W$ is formal (as the self-intersection of the zero section in a vector bundle). Theorem 1.4 shows that the line bundle $\mathcal{L}$ on $W$ associated with this twisted intersection problem is $\left(F_{*} \Omega_{X}^{*}\right)^{\vee}$. This gives a geometric meaning to the obstruction $\alpha$ to the formality of $F_{*} \Omega_{X}^{*}$ : it is precisely the class of $\mathcal{L}$ in $\operatorname{Pic}(W)$. Indeed, in Deligne-Illusie's paper it is shown that $\alpha$ lies in $H^{2}\left(X^{\prime}, T_{X^{\prime}}\right)$, and we will argue that this group is a direct summand in $\operatorname{Pic}(W)$, thus confirming the geometric description above.

One can ask for a criterion, for an arbitrary twisted derived intersection problem, that will imply the triviality of the associated line bundle $\mathcal{L}$. We give such a criterion in Theorem 4.4. For a self-intersection, this result is similar in nature to Theorem 1.2; for an arbitrary intersection, it is a generalization to Azumaya spaces of the main result of [ACH14].

For the Deligne-Illusie application, the main condition of Theorem 4.4 that needs to be satisfied in order to conclude the formality of $F_{*} \Omega_{X}^{*}$ is for the trivial bundle of $X^{\prime}$ to extend to the first infinitesimal neighborhood of the embedding $h$. This extension problem needs to be understood correctly in the context of maps of Azumaya spaces. We will argue (see the proof of 


\section{DERIVED INTERSECTIONS AND THE HODGE THEOREM}

Theorem 4.8) that the trivial bundle extends if and only if the Azumaya algebra $D$ splits on the first infinitesimal neighborhood of the zero section in $T^{*} X^{\prime}$.

In this context, we can state the second result of this paper.

Theorem 1.6. Let $X$ be a smooth scheme over a perfect field $\mathbf{k}$ of characteristic $p>\operatorname{dim} X$. Then the following five statements are equivalent:

(i) The scheme $X$ lifts to $W_{2}(\mathbf{k})$.

(ii) The algebra $D$ splits on the first infinitesimal neighborhood of $X^{\prime}$ in $T^{*} X^{\prime}$.

(iii) The trivial bundle $\mathscr{O}_{X^{\prime}}$ lifts to the first infinitesimal neighborhood of the embedding $h$.

(iv) The associated line bundle $\mathcal{L} \in \operatorname{Pic}(W)$ is trivial.

(v) The complex $F_{*} \Omega_{X}^{\bullet}$ is formal in $\mathbf{D}\left(X^{\prime}\right)$.

Remark 1.7. Some of the implications above are not new. The equivalence of statements (i) and (v) is the original result of Deligne and Illusie [DI87]. The implication (i) $\Rightarrow$ (ii) was proven by Ogus and Vologodsky [OV07], where a splitting module is explicitly constructed. The implications (ii) $\Rightarrow$ (iii) $\Rightarrow$ (iv) $\Rightarrow$ (v) $\Rightarrow$ (ii) are parts of Theorem 4.4 and give a geometric interpretation of Deligne and Illusie's result.

The twisted de Rham complex. There is a generalization of the de Rham theorem that is of interest in the study of singularity theory and in the theory of matrix factorizations, and which involves twisted de Rham complexes. This generalization was investigated by Barannikov and Kontsevich [BK98] and Sabbah [Sab99], and in the remainder of this introduction we discuss how some of their results can also be understood as formality results for derived intersections.

Let $X$ be a smooth quasi-projective scheme over $\mathbb{C}$, with a map $f: X \rightarrow \mathbb{A}^{1}$ such that its critical locus is proper over $\mathbb{A}^{1}$. These data give rise to two complexes defined as

$$
\Omega_{X, d-\wedge d f}^{\bullet}: \quad 0 \rightarrow \mathcal{O}_{X} \stackrel{d-\wedge d f}{\longrightarrow} \Omega_{X}^{1} \stackrel{d-\wedge d f}{\longrightarrow} \cdots \stackrel{d-\wedge d f}{\longrightarrow} \Omega_{X}^{n} \rightarrow 0,
$$

and

$$
\Omega_{X, \wedge d f}^{\bullet}: \quad 0 \rightarrow \mathcal{O}_{X} \stackrel{\wedge d f}{\longrightarrow} \Omega_{X}^{1} \stackrel{\wedge d f}{\longrightarrow} \cdots \stackrel{\wedge d f}{\longrightarrow} \Omega_{X}^{n} \rightarrow 0 .
$$

The following theorem, due to Barannikov-Kontsevich [BK98] and Sabbah [Sab99], relates the hypercohomology of these two complexes.

THEOREM 1.8. The hypercohomology spaces of the two complexes defined above are of the same (finite) dimensions.

Note that the de Rham theorem is the particular case of the above result when $f=0$. In [Sab99], Sabbah asked for a purely algebraic proof of the result of Barannikov-Kontsevich. Such a proof was given by Ogus and Vologodsky [OV07], again using positive characteristic methods.

We can ask again if these results can be rephrased as formality statements for derived intersections, and in what follows we shall give a generalization of Theorem 1.4 and a partial version of the equivalence in Theorem 1.6.

Consider a smooth scheme $X$ over a perfect field $\mathbf{k}$ of positive characteristic $p$, with a regular function $f: X \rightarrow \mathbb{A}^{1}$. The map $f$ provides a morphism $f^{\prime}: X^{\prime} \rightarrow \mathbb{A}^{1}$. We denote the graph of $d\left(f^{\prime}\right)$ 


\section{Arinkin, A. CăLdăraru And M. Hablicsek}

in $T^{*} X^{\prime}$ by $X_{f}^{\prime}$, and it is easy to see (Remark 5.7) that $D$ splits on the section $X_{f}^{\prime}$ just as it did on the zero section $X^{\prime}$, giving rise to an isomorphism

$$
n:\left(X_{f}^{\prime}, \mathscr{O}_{X_{f}^{\prime}}\right) \rightarrow\left(X_{f}^{\prime},\left.D\right|_{X_{f}^{\prime}}\right) .
$$

The following result generalizes Theorem 1.4.

Theorem 1.9. Consider the embeddings $i: X^{\prime} \hookrightarrow T^{*} X^{\prime}$ and $j: X_{f}^{\prime} \hookrightarrow T^{*} X^{\prime}$ and the composite morphisms

$$
i^{\prime}:\left(X^{\prime}, \mathcal{O}_{X^{\prime}}\right) \stackrel{m}{\longrightarrow}\left(X^{\prime},\left.D\right|_{X^{\prime}}\right) \stackrel{i_{D}}{\longrightarrow}\left(T^{*} X^{\prime}, D\right)
$$

and

$$
j^{\prime}:\left(X_{f}^{\prime}, \mathcal{O}_{X_{f}^{\prime}}\right) \stackrel{n}{\rightarrow}\left(X_{f}^{\prime},\left.D\right|_{X_{f}^{\prime}}\right) \stackrel{j_{D}}{\longrightarrow}\left(T^{*} X^{\prime}, D\right) .
$$

Then we have isomorphisms in $\mathbf{D}\left(X^{\prime}\right)$

$$
\begin{aligned}
\Omega_{X^{\prime}, \wedge d f^{\prime}} & \cong i^{!} j_{*} \mathcal{O}_{X_{f}^{\prime}}, \\
F_{*} \Omega_{X, d-\wedge d f}^{\bullet} & \cong i^{\prime !} j_{*}^{\prime} \mathcal{O}_{X_{f}^{\prime}} .
\end{aligned}
$$

Note that we are precisely in the general context of twisted derived intersections set up in Remark 1.5: we are studying the derived intersections $W$ and $\bar{W}$ of $X^{\prime}$ and $X_{f}^{\prime}$ inside $S=T^{*} X^{\prime}$ and $\bar{S}=\left(T^{*} X^{\prime}, D\right)$, respectively. Indeed, the above theorem shows that $\mathscr{O}_{W}$ and $\mathscr{O}_{\bar{W}}$ are precisely the duals of $\Omega_{X^{\prime}, \wedge d f^{\prime}}$ and $F_{*} \Omega_{X, d-\wedge d f}^{\cdot}$, respectively.

Now, we can apply the full power of Theorem 4.4 to prove the following partial version of the Barannikov-Kontsevich claim.

THEOREM 1.10. Let $X$ be a smooth scheme over a field of characteristic zero. Let $f$ be a regular function on $X$ such that the map $f: X \rightarrow \mathbb{A}^{1}$ is proper, and assume that the following two conditions hold:

(a) The critical locus $Z=$ Crit $f$ is scheme-theoretically smooth.

(b) The embedding $p: Z \hookrightarrow X$ is split to first order; that is, the short exact sequence

$$
\left.0 \rightarrow T_{Z} \rightarrow T_{X}\right|_{Z} \rightarrow N_{Z / X} \rightarrow 0
$$

splits.

Then the complexes $F_{*} \Omega_{X, d-\wedge d f}^{\bullet}$ and $\Omega_{X^{\prime}, \wedge d f^{\prime}}$ are both formal, and there exist isomorphisms

$$
\mathbf{R}^{i} \Gamma\left(X, \Omega_{X, d-\wedge d f}^{\bullet}\right) \cong \mathbf{R}^{i} \Gamma\left(X, \Omega_{X, \wedge d f}^{\bullet}\right) \cong \bigoplus_{i-c=p+q} H^{p}\left(Z, \Omega_{Z}^{q} \otimes \omega\right)
$$

where $c$ denotes the codimension of $Z$ in $X$ and $\omega$ denotes the relative dualizing complex of the embedding $Z \hookrightarrow X$.

The paper is organized as follows. In Section 2, we collect basic facts about schemes over fields of positive characteristic and about the sheaf of crystalline differential operators. We follow with Section 3, where we set up the 2-category of Azumaya spaces. In Section 4, we discuss the general problem of twisted derived intersections and we prove Theorem 4.4. We conclude the paper with a final section where we apply the general results we have developed to the study of the formality problem for de Rham complexes. In particular, we prove Theorems 1.4, 1.9, and 1.10. 


\section{DERIVED INTERSECTIONS AND THE HODGE THEOREM}

\section{Background on schemes over fields of positive characteristics}

Let $X$ be a smooth scheme over a perfect field $\mathbf{k}$ of characteristic $p>0$. The classical Frobenius morphism on $X$ acts trivially on the underlying topological space but acts by the $p$ th power map on the structure sheaf. The Frobenius twist $X^{\prime}$ of $X$ is obtained by taking the base change of $X$ by the classical Frobenius map Spec $\mathbf{k} \rightarrow$ Speck. Since the classical Frobenius morphisms on $X$ and on Spec $\mathbf{k}$ commute with the structure map $X \rightarrow$ Spec $\mathbf{k}$, we obtain a commutative diagram

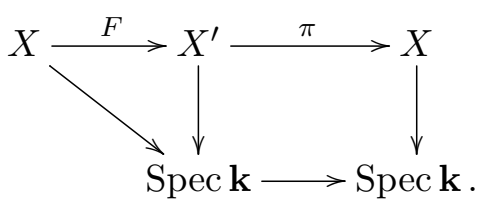

The map $F: X \rightarrow X^{\prime}$ in this diagram is called the relative Frobenius morphism. By construction, it is a map of schemes over $\mathbf{k}$.

Since $\mathbf{k}$ is a perfect field, the map Spec $\mathbf{k} \rightarrow$ Spec $\mathbf{k}$ is an isomorphism. Therefore, the map $\pi: X^{\prime} \rightarrow X$ is an isomorphism of abstract schemes. However, this isomorphism is not a morphism of schemes over $\mathbf{k}$.

The algebraic de Rham complex of $X$ is defined as the complex

$$
\Omega_{X}^{\bullet}=0 \rightarrow \Omega_{X}^{0} \stackrel{d}{\rightarrow} \Omega_{X}^{1} \stackrel{d}{\rightarrow} \cdots \rightarrow 0,
$$

where $d$ is the usual de Rham differential on algebraic forms. The algebraic de Rham cohomology of $X$ is defined as

$$
H_{\mathrm{d} R}^{*}(X)=R^{*} \Gamma\left(X, \Omega_{X}^{\bullet}\right) .
$$

The Hodge-de Rham spectral sequence

$$
{ }^{1} E^{p q}=H^{p}\left(X, \Omega_{X}^{q}\right) \Rightarrow H_{\mathrm{d} R}^{p+q}(X)
$$

arises from the 'stupid' filtration of $\Omega_{X}^{*}$, whose associated graded terms are $\Omega_{X}^{q}$.

It is important to note that while the sheaves in the complex $\Omega_{X}^{\circ}$ are coherent $\mathscr{O}_{X}$-modules, the differentials are not $\mathscr{O}_{X}$-linear, so we shall only consider the above complex as a complex of sheaves of abelian groups. On the other hand, the equality

$$
d\left(f^{p} g\right)=d\left(f^{p}\right) \cdot g+f^{p} \cdot d(g)=p f^{p-1} d f \cdot g+f^{p} \cdot d(g)
$$

implies that $F_{*} \Omega_{X}^{*}$ is a complex of coherent $\mathcal{O}_{X^{\prime}}$-modules (the differentials are now $\mathcal{O}_{X^{\prime}}$-linear).

The main result of Deligne-Illusie, Theorem 1.1, asserts that if $X$ is proper over $\mathbf{k}$ and lifts to $W_{2}(\mathbf{k})$, then the Hodge-de Rham spectral sequence degenerates at ${ }^{1} E$. This follows easily from the central statement that under the liftability assumption to $W_{2}(\mathbf{k})$, the complex $F_{*} \Omega_{X}^{*}$ is formal. We describe this implication.

A well-known result of Cartier describes the cohomology sheaves of $F_{*} \Omega_{X}^{\circ}$ for any $X$ smooth over $k$ :

$$
\mathcal{H}^{i}\left(X^{\prime}, F_{*} \Omega_{X}^{*}\right) \cong \Omega_{X^{\prime}}^{i} .
$$

If $X$ lifts to $W_{2}(\mathbf{k})$, then $F_{*} \Omega_{X}^{\bullet}$ is formal; therefore,

$$
F_{*} \Omega_{X}^{\cdot} \cong \bigoplus_{i=0}^{\operatorname{dim} X} \Omega_{X^{\prime}}^{i}[-i] .
$$




\section{Arinkin, A. Căldăraru And M. Hablicsek}

Taking hypercohomology of both sides, we obtain an isomorphism

$$
H_{\mathrm{d} R}^{i}(X) \cong \bigoplus_{i=p+q} H^{p}\left(X^{\prime}, \Omega_{X^{\prime}}^{q}\right) \cong \bigoplus_{i=p+q} H^{p}\left(X, \Omega_{X}^{q}\right)
$$

where the latter isomorphism is a consequence of the fact that $X$ and $X^{\prime}$ are isomorphic as abstract schemes. The assumption that $X$ is proper over $k$ implies that the vector spaces $H^{p}\left(X, \Omega_{X}^{q}\right)$ are finite dimensional, and the degeneration at ${ }^{1} E$ of the Hodge-de Rham spectral sequence follows by comparing dimensions.

Another key ingredient in what follows is the sheaf of crystalline differential operators (see [BMR08] for a detailed description). We emphasize that we do not work with Grothendieck's ring of differential operators $\mathbb{D}$. Instead, we use the ring of crystalline differential operators $D_{X}$, which is the quasi-coherent sheaf of algebras over $X$ defined as the universal enveloping algebra of the Lie algebroid of the tangent bundle $T_{X}$. Explicitly, it is defined locally as the $k$-algebra generated by $T_{X}$ and $\mathcal{O}_{X}$ subject to the relations

- $f \cdot \partial=f \partial$ for all $f \in \mathcal{O}_{X}$ and $\partial \in T_{X}$,

- $\partial_{1} \cdot \partial_{2}-\partial_{2} \cdot \partial_{1}=\left[\partial_{1}, \partial_{2}\right]$ for all $\partial_{i} \in T_{X}$, and

- $\partial \cdot f-f \cdot \partial=\partial(f)$ for all $f \in \mathcal{O}_{X}$ and $\partial \in T_{X}$.

In the case of $X=\mathbb{A}^{n}=\operatorname{Spec} k\left[\underline{x}_{i}\right]$, this construction produces the Weyl algebra $D_{X}=k\left\langle\underline{x}_{i}, \underline{\partial}_{i}\right\rangle$.

The main difference between $D_{X}$ and $\mathbb{D}$ is best understood in the case where $X=\mathbb{A}^{1}=$ Spec $k[x]$. If we let $\partial=\partial / \partial x$, then in the ring $\mathbb{D}$, the operator $\partial^{p}$ is zero because its action on functions is trivial. In the ring $D_{X}$, the operator $\partial^{p}$ is non-trivial. On the other hand, in $\mathbb{D}$ there is an element of the form $\partial^{p} / p$ ! which does not exist in $D_{X}$.

Explicitly, a $D_{X}$-module $F$ is an $\mathscr{O}_{X}$-module together with an action of the Lie algebroid $T_{X}$. Such an action can be represented as an integrable connection

$$
\nabla=\nabla_{F}: F \rightarrow \Omega_{X}^{1} \otimes F .
$$

In local coordinates $\left\{x_{1}, \ldots, x_{n}\right\}$, the connection is given by

$$
\nabla(f)=\sum_{i=1}^{n} d x_{i} \otimes \frac{\partial}{\partial x_{i}}(f) .
$$

Remark 2.1. The sheaf $\mathscr{O}_{X}$ is naturally a (left) $D_{X}$-module. This $D_{X^{-}}$-module admits a Koszul resolution of the form

$$
0 \rightarrow D_{X} \otimes\left(\wedge^{\operatorname{dim} X} T_{X}\right) \rightarrow \cdots \rightarrow D_{X} \otimes T_{X} \rightarrow D_{X} \rightarrow \mathscr{O}_{X} \rightarrow 0 .
$$

The tensor products are over $\mathscr{O}_{X}$, with $\mathscr{O}_{X}$ acting on $D_{X}$ on the right. This is a resolution of $\mathscr{O}_{X}$ by locally free $D_{X}$-modules; therefore, it can be used to compute local Ext groups Accordingly, if $F$ is any $D_{X}$-module, the derived sheaf of homomorphisms $\underline{\mathbf{H o m}}_{\left(X, D_{X}\right)}\left(\mathscr{O}_{X}, F\right)$ is represented by the de Rham complex of $F$ :

$$
\mathrm{d} R(F):=0 \rightarrow \Omega_{X}^{0} \otimes F \stackrel{d_{F}}{\longrightarrow} \Omega_{X}^{1} \otimes F \stackrel{d_{F}}{\rightarrow} \cdots,
$$

where the differentials $d_{F}: \Omega_{X}^{j} \otimes F \rightarrow \Omega_{X}^{j+1} \otimes F$ are given by

$$
d_{F}(\omega \otimes f)=d(\omega) \otimes f+(-1)^{j} \omega \wedge \nabla(f) .
$$

In particular, if $F=\mathscr{O}_{X}$, the de Rham complex $\mathrm{d} R\left(\mathscr{O}_{X}\right)$ is the (algebraic) de Rham complex of $X$. 


\section{Derived INTERSECTIONS AND THE Hodge THEOREM}

Remark 2.2. The following general properties of the ring of crystalline differential operators are well known; see [BMR08]. A priori, $D_{X}$ is constructed as a sheaf of (left) $\mathscr{O}_{X}$-modules on $X$. However, the center of $D_{X}$ can be canonically identified with the structure sheaf of the cotangent bundle $T^{*} X^{\prime}$ of $X^{\prime}$, the Frobenius twist of $X$. We review this identification.

Since the map $\pi: X^{\prime} \rightarrow X$ is an isomorphism, it induces an isomorphism of sheaves $\pi_{*}$ : $T_{X^{\prime}} \rightarrow T_{X}$, which in local coordinates can be described as

$$
\pi_{*}\left(\frac{\partial}{\partial\left(x^{p}\right)}\right)=\frac{\partial}{\partial x} .
$$

(Obviously, the map is not $k$-linear.) For any vector field $\partial \in T_{X}$, its $p$ th power $\partial^{p} \in D_{X}$ acts again as a derivation on the functions on $X$. Therefore, it can be regarded as another vector field, denoted by $\partial^{[p]}$. In Lie-theoretic terms, vector fields on $X$ form a $p$-restricted Lie algebra.

Consider the map $\iota: T_{X^{\prime}} \rightarrow D_{X}$ obtained as the composite

$$
T_{X^{\prime}} \stackrel{\pi_{*}}{\longrightarrow} T_{X} \stackrel{\partial \mapsto \partial^{p}-\partial^{[p]}}{\longrightarrow} D_{X}
$$

It is well known [BMR08, Lemma 1.3.1] that $\iota$ is $\mathscr{O}_{X^{\prime}}$-linear, and the image is in the center of $D_{X}$. Since $f^{p}$ lies in the center of $D_{X}$ for $f \in \mathcal{O}_{X}$, the map $\iota$ extends to a homomorphism $\bar{\iota}: \mathscr{O}_{T^{*} X^{\prime}} \rightarrow Z\left(D_{X}\right)$. One can check that this map is an isomorphism [BMR08, Lemma 1.3.2].

Remark 2.3. As a consequence, we can regard $D_{X}$ as a sheaf of algebras on $T^{*} X^{\prime}$ instead of on $X$. To avoid confusion we will denote $D_{X}$ by $D$ when it is regarded as a sheaf on $T^{*} X^{\prime}$. The projection to the zero section $\phi: T^{*} X^{\prime} \rightarrow X^{\prime}$ identifies $D$ and $D_{X}$; we have $\phi_{*} D=F_{*} D_{X}$.

Remark 2.4. The algebra $D_{X}$ is an Azumaya algebra over its center, and so $D$ is a sheaf of Azumaya algebras over $T^{*} X^{\prime}$. The restriction of $D$ to the zero section, $\left.D\right|_{X^{\prime}}=i^{*} D$, is a split Azumaya algebra, with splitting module $F_{*} \mathcal{O}_{X}$. In other words, $\left.D\right|_{X^{\prime}}=\underline{\text { End }}_{X^{\prime}}\left(F_{*} \mathcal{O}_{X}\right)$. Therefore, $\mathcal{O}_{X^{\prime}}$ and $\left.D\right|_{X^{\prime}}$ are Morita equivalent: the functors

$$
m_{*}: \mathfrak{M o d}-\mathcal{O}_{X^{\prime}} \rightarrow \mathfrak{M o d}-\left.D\right|_{X^{\prime}}, \quad M \mapsto M \otimes F_{*} \mathcal{O}_{X}
$$

and

$$
m^{*}: \operatorname{Mod}-\left.D\right|_{X^{\prime}} \rightarrow \mathfrak{M o d}-\mathcal{O}_{X^{\prime}}, \quad N \mapsto \underline{\operatorname{Hom}}_{\left.D\right|_{X^{\prime}}}\left(F_{*} \mathcal{O}_{X}, N\right)
$$

are inverse to each other. These functors give rise to an equivalence between the corresponding derived categories $\mathbf{D}\left(X^{\prime}, D_{X^{\prime}}\right)$ and $\mathbf{D}\left(X^{\prime}\right)$. We shall denote the induced functors on derived categories by $m_{*}$ and $m^{*}$ as well.

\section{Azumaya schemes}

Our results are naturally formulated in the framework of schemes equipped with Azumaya algebras. Such objects are called 'twisted spaces' in [Căl00], but we prefer the more descriptive name Azumaya schemes. It is worth noting that one can pass from Azumaya schemes to the corresponding $\mathbb{G}_{m}$-gerbes (see Remark 3.8); the results below can be easily restated in the language of gerbes. One advantage of Azumaya algebras is that they are somewhat more explicit than gerbes.

Definition 3.1. An Azumaya scheme is a pair $(S, \mathscr{A})$ where $S$ is a scheme and $\mathscr{A}$ is a (coherent) sheaf of Azumaya algebras over $S$. Given a property of schemes that is local in the étale topology, we say that $(S, \mathscr{A})$ has this property if and only if $S$ does. 


\section{Arinkin, A. CăLdăRARU And M. Hablicsek}

Suppose that $(S, \mathscr{A})$ is an Azumaya scheme and $f: S^{\prime} \rightarrow S$ is a morphism from another scheme $S^{\prime}$ to $S$. Then the pull-back $f^{*} \mathscr{A}$ is an Azumaya algebra over $S^{\prime}$. We could consider the category whose objects are Azumaya schemes $(S, \mathscr{A})$ and where morphisms $\left(S^{\prime}, \mathscr{A}^{\prime}\right) \rightarrow(S, \mathscr{A})$ are pairs

$$
\left(f: S^{\prime} \rightarrow S, \quad \phi: \quad f^{*} \mathscr{A} \simeq \mathscr{A}^{\prime}\right) .
$$

However, such a 'strict' category of Azumaya schemes is too restrictive from the viewpoint of Azumaya algebras: one should work with Morita equivalences instead of isomorphisms. This leads to the following definition.

Definition 3.2. A morphism (or, more precisely, a 1-morphism)

$$
\left(S^{\prime}, \mathscr{A}^{\prime}\right) \rightarrow(S, \mathscr{A})
$$

of Azumaya schemes is a pair $(f, E)$, where $f: S^{\prime} \rightarrow S$ is a morphism of schemes and $E$ is an $f^{*}(\mathscr{A})^{\text {op }} \otimes \mathscr{A}^{\prime}$-module that provides a Morita equivalence between the Azumaya algebras $\mathscr{A}^{\prime}$ and $f^{*} \mathscr{A}$.

Given two 1-morphisms $\left(f_{1}, E_{1}\right)$ and $\left(f_{2}, E_{2}\right)$ between Azumaya schemes $\left(S^{\prime}, \mathscr{A}^{\prime}\right)$ and $(S, \mathscr{A})$, there exist 2-morphisms between these 1-morphisms only if $f_{1}=f_{2}$. In the case $f_{1}=f_{2}$, such 2-morphisms are isomorphisms between the $f_{1}^{*}(\mathscr{A})^{\mathrm{op}} \otimes \mathscr{A}^{\prime}$-modules $E_{1}$ and $E_{2}$.

Remark 3.3. The condition that $E$ provides a Morita equivalence between the Azumaya algebras $\mathscr{A}^{\prime}$ and $f^{*} \mathscr{A}$ implies that $E$ is a vector bundle on $S^{\prime}$ whose rank at $y^{\prime} \in S$ is the product of the ranks of $\mathscr{A}^{\prime}$ at $y^{\prime}$ and $\mathscr{A}$ at $f\left(y^{\prime}\right)$. (The rank of an Azumaya algebra $\mathscr{A}$ is $n$ if its rank as an $\mathscr{O}_{S}$-module is $n^{2}$.)

It is easy to see that Azumaya schemes form a 2-category with respect to the natural composition of 1-morphisms. The category is equipped with a forgetful functor to the category of schemes, which sends the Azumaya scheme $(S, \mathscr{A})$ to $S$. We think of the Azumaya scheme $(S, \mathscr{A})$ as a 'twisting' of the scheme $S$; accordingly, we call the forgetful functor the untwisting.

Conversely, for any scheme $S$ we have the 'untwisted' Azumaya scheme $\left(S, \mathscr{O}_{S}\right)$; if no confusion is likely, we still denote this Azumaya scheme by $S$. Note, however, that the corresponding functor from the category of schemes to the 2-category of Azumaya schemes is not fully faithful (see Example 3.4).

From now on, we suppose that all our Azumaya schemes are of finite type over a fixed ground field; this ensures that direct and inverse image functors are defined and well behaved. A quasicoherent sheaf on an Azumaya scheme $(S, \mathscr{A})$ is a sheaf of $\mathscr{A}$-modules that is quasi-coherent as an $\mathscr{O}_{S}$-module. Quasi-coherent sheaves on $(S, \mathscr{A})$ form an abelian category with enough injectives; denote the corresponding derived category by $\mathbf{D}(S, \mathscr{A})$. Of course, if $\left(S, \mathscr{O}_{S}\right)$ is the untwisted Azumaya scheme, the category $\mathbf{D}\left(S, \mathscr{O}_{S}\right)$ is the usual derived category $\mathbf{D}(S)$.

Let $\phi=(f, E)$ be a morphism of Azumaya schemes $\left(S^{\prime}, \mathscr{A}^{\prime}\right) \rightarrow(S, \mathscr{A})$. The direct image functor $f_{*}: \mathbf{D}\left(S^{\prime}\right) \rightarrow \mathbf{D}(S)$ and the inverse image functor $f^{*}: \mathbf{D}(S) \rightarrow \mathbf{D}\left(S^{\prime}\right)$ are naturally upgraded to functors $f_{*}: \mathbf{D}\left(S^{\prime}, f^{*} \mathscr{A}\right) \rightarrow \mathbf{D}(S, \mathscr{A})$ and $f^{*}: \mathbf{D}(S, \mathscr{A}) \rightarrow \mathbf{D}\left(S^{\prime}, \mathscr{A}^{\prime}\right)$. Composing them with the Morita equivalence

$$
\mathbf{D}\left(S^{\prime}, \mathscr{A}^{\prime}\right) \simeq \mathbf{D}\left(S^{\prime}, f^{*} \mathscr{A}\right)
$$

given by $E$, we obtain the direct image functor

$$
\phi_{*}: \mathbf{D}\left(S^{\prime}, \mathscr{A}^{\prime}\right) t o \mathbf{D}(S, \mathscr{A}), \quad M^{\prime} \mapsto f_{*}\left(E^{\vee} \otimes_{\mathscr{A}^{\prime}} M^{\prime}\right)
$$




\section{Derived INTERSECTIONS AND THE Hodge THEOREM}

and the inverse image functor

$$
\phi^{*}: \mathbf{D}(S, \mathscr{A}) \rightarrow \mathbf{D}\left(S^{\prime}, \mathscr{A}^{\prime}\right), \quad M \mapsto E \otimes_{f^{*} \mathscr{A}} f^{*}(M) .
$$

It is easy to see that $\phi^{*}$ is the left adjoint of $\phi_{*}$.

Let us now consider the 'extraordinary' inverse image functor. Let $\phi$ be as above. Generally speaking, the inverse image functor $f^{!}$is defined only on bounded below categories

$$
f^{!}: \mathbf{D}^{+}(S) \rightarrow \mathbf{D}^{+}\left(S^{\prime}\right)
$$

accordingly, we obtain the inverse image functor

$$
\phi^{!}: \mathbf{D}^{+}(S, \mathscr{A}) \rightarrow \mathbf{D}^{+}\left(S^{\prime}, \mathscr{A}^{\prime}\right), \quad M \mapsto E \otimes_{f^{*} \mathscr{A}} f^{!}(M)
$$

between the bounded below categories of the Azumaya schemes. If $f$ is Gorenstein (or, more generally, if the relative dualizing complex of $S^{\prime}$ over $S$ is perfect), the functor $f^{!}$can be extended to the unbounded categories, and then $\phi^{!}$also extends to the inverse image functor

$$
\phi^{!}: \mathbf{D}(S, \mathscr{A}) \rightarrow \mathbf{D}\left(S^{\prime}, \mathscr{A}^{\prime}\right) .
$$

For instance, this is always the case for morphisms between smooth Azumaya schemes.

If the map $\phi$ is proper (that is, if $f$ is proper), then $\phi^{!}$is the right adjoint of $\phi_{*}$.

Example 3.4. Let $(S, \mathscr{A})$ be an Azumaya scheme. The identity 1-morphism of $(S, \mathscr{A})$ is the pair $\left(\operatorname{id}_{S}, \mathscr{A}\right)$, where we view $\mathscr{A}$ as an $\mathscr{A}^{\mathrm{op}} \otimes \mathscr{A}$-module in the natural way. If $\mathcal{L}$ is a line bundle on the (untwisted) scheme $S$, the pair

$$
\phi_{\mathcal{L}}=\left(\operatorname{id}_{S}, \mathcal{L} \otimes \mathscr{A}\right)
$$

is a 1-automorphism of $(S, \mathscr{A})$ as well; in this way, we obtain all 1-automorphisms of $(S, \mathscr{A})$ that act trivially on its 'untwisting' $S$. In particular, even if $\mathscr{A}=\mathscr{O}_{S}$ and $(S, \mathscr{A})$ is the usual 'untwisted' scheme, it acquires some 'twisted' automorphisms $\left(\phi_{\mathcal{L}}\right)$ when considered as an Azumaya scheme.

The pull-back and push-forward functors for the morphism $\phi_{\mathcal{L}}$ are then given by

$$
\begin{aligned}
\phi^{*}=\phi^{!}: \mathbf{D}(S, \mathscr{A}) & \rightarrow \mathbf{D}(S, \mathscr{A}), & \phi_{*}: \mathbf{D}(S, \mathscr{A}) & \rightarrow \mathbf{D}(S, \mathscr{A}), \\
M & \mapsto M \otimes \mathcal{L}, & M & \mapsto M \otimes \mathcal{L}^{-1} .
\end{aligned}
$$

Example 3.5. Suppose that we are given three Azumaya schemes $\bar{X}=\left(X, \mathscr{A}_{X}\right), \bar{Y}=\left(Y, \mathscr{A}_{Y}\right)$, and $\bar{S}=(S, \mathscr{B})$ and morphisms

$$
\phi_{X}=\left(f_{X}, E_{X}\right): \bar{X} \rightarrow \bar{S}, \quad \phi_{Y}=\left(f_{Y}, E_{Y}\right): \bar{Y} \rightarrow \bar{S} .
$$

The fibered product $W:=X \times_{S} Y$ is equipped with a natural Azumaya algebra $\mathscr{A}$, making it into an Azumaya space $\bar{W}=(W, \mathscr{A})$. Explicitly,

$$
p_{1}^{*}\left(E_{X}\right) \otimes p_{2}^{*}\left(E_{Y}\right)
$$

is a module over

$$
p_{1}^{*}\left(\mathscr{A}_{X}\right) \otimes p_{2}^{*}\left(\mathscr{A}_{Y}\right) \otimes\left(p^{*}(\mathscr{B})^{\otimes 2}\right)^{\mathrm{op}},
$$

and we let $\mathscr{A}$ be the algebra of its endomorphisms as a $\left(p^{*}(\mathscr{B})^{\otimes 2}\right)^{\mathrm{op}}$-module. Here $p_{1}, p_{2}$, and $p$ are the projections of $W$ onto $X, Y$, and $S$, respectively.

The four Azumaya algebras $\mathscr{A}, p_{1}^{*}\left(\mathscr{A}_{X}\right), p_{2}^{*}\left(\mathscr{A}_{Y}\right)$, and $p^{*}(\mathscr{B})$ are naturally Morita equivalent; 


\section{Arinkin, A. CăLd ĂRARU AND M. Hablicsek}

this gives a commutative square of Azumaya schemes

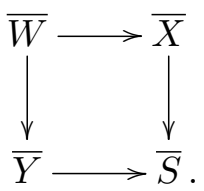

It is not hard to see that this square is 1-Cartesian, so that $\bar{W}$ is the fiber product of $\bar{X}$ and $\bar{Y}$ over $\bar{S}$.

A particular case of this construction is when $\mathscr{A}_{X}=f_{X}^{*} \mathscr{B}$ and $\mathscr{A}_{Y}=f_{Y}^{*} \mathscr{B}$. In this case, the algebra $\mathscr{A}$ can naturally be identified with $p^{*} \mathscr{B}$.

Remark 3.6. Note that the 2-category of Azumaya schemes does not have a final object. For this reason, while fiber products exist, Cartesian products do not.

EXAmple 3.7. Now, suppose in addition that the two Azumaya schemes $\bar{X}$ and $\bar{Y}$ are untwisted, so that $\mathscr{A}_{X}=\mathscr{O}_{X}$ and $\mathscr{A}_{Y}=\mathscr{O}_{Y}$. In this case, the Azumaya scheme $(W, \mathscr{A})$ is also split. However, it carries two natural splittings: one of them compatible with the splitting of the Azumaya scheme $\bar{X}$ and the other compatible with the splitting of $\bar{Y}$. The difference between the two splittings is a line bundle $\mathcal{L}$ on $S$. Explicitly,

$$
\mathcal{L}=\underline{\operatorname{Hom}}_{p^{*}(\mathscr{B})^{\mathrm{op}}}\left(p_{1}^{*}\left(E_{1}\right), p_{2}^{*}\left(E_{2}\right)\right)
$$

measures the discrepancy between the splitting of the pull-backs $f_{X}^{*}(\mathscr{B})$ and $f_{Y}^{*}(\mathscr{B})$.

In this way, we obtain a commutative diagram

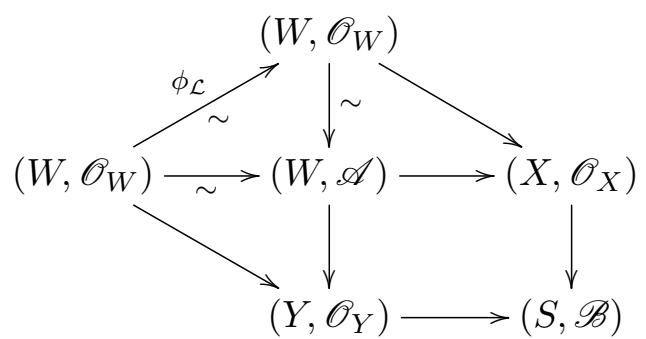

of Azumaya schemes. The morphism $\phi_{\mathcal{L}}$ is constructed in Example 3.4.

All of the above constructions admit differential-graded analogues. In particular, in the situation of Example 3.5 we may consider the derived fiber product of the Azumaya schemes. Specifically, the derived fiber product of $X$ and $Y$ over $S$ is naturally equipped with a sheaf of Azumaya algebras, and we thus obtain an Azumaya $d g$-scheme $W^{R}$.

This is the only way in which Azumaya dg-schemes appear in this paper. In fact, for our purposes the most important situation is as in Example 3.7: the Azumaya schemes $\bar{X}$ and $\bar{Y}$ are non-twisted, and thus their derived fiber product $W^{R}$ is split (that is, 1-isomorphic to an untwisted dg-scheme); however, there are two splittings that differ by a line bundle on the dgscheme $W^{R}$. For details, see Section 4 .

Remark 3.8. It is well known that an Azumaya algebra on a scheme $S$ yields a class in the Brauer group $H^{2}\left(S, \mathscr{O}_{S}^{\times}\right)$, which can be interpreted geometrically as a $\mathbb{G}_{m}$-gerbe on $S$. (Here, $\mathbb{G}_{m}$ is the multiplicative group.)

Explicitly, to an Azumaya scheme $(S, \mathscr{A})$, we assign the stack $\operatorname{Spl}(\mathscr{A})$ such that morphisms $T \rightarrow \operatorname{Spl}(\mathscr{A})$ from a test scheme $T$ are pairs $(f, E)$, where $f: T \rightarrow S$ and $E$ is the splitting of 


\section{DERIVED INTERSECTIONS AND THE HODGE THEOREM}

the Azumaya algebra $f^{*} \mathscr{A}$. Equivalently, $\left(f, E^{\vee}\right)$ is a morphism of Azumaya schemes $\left(T, \mathscr{O}_{T}\right) \rightarrow$ $(S, \mathscr{A})$.

It is clear that $\operatorname{Spl}(\mathscr{A})$ is equipped with a natural morphism to $S$ and that this morphism turns $\operatorname{Spl}(\mathscr{A})$ into a $\mathbb{G}_{m}$-gerbe over $S$. In this way, we obtain a fully faithful embedding of the 2-category of Azumaya schemes into the 2-category of $\mathbb{G}_{m}$-gerbes.

Since $\operatorname{Spl}(\mathscr{A})$ is a $\mathbb{G}_{m}$-gerbe, the category $\mathbf{D}(\operatorname{Spl}(\mathscr{A}))$ decomposes into direct summands indexed by the characters of $\mathbb{G}_{m}$. The summand corresponding to the tautological character is identified with $\mathbf{D}(S, \mathscr{A})$. This identification agrees with the pull-back and push-forward functors for a morphism between Azumaya schemes.

\section{Twisted derived intersections}

In this section, we discuss derived intersections in twisted spaces, generalizing the results of [AC12] and [ACH14]. We argue that there is a natural twisted Hochschild-Kostant-Rosenberg (HKR) class with properties similar to those of the usual one. The most important observation is that, under certain assumptions, the twisted derived intersection differs only by a line bundle from the untwisted one.

We begin our discussion with the general framework for the derived intersection problems we study.

Let $S$ be a smooth variety, and let $X$ and $Y$ be smooth subvarieties of $S$, with corresponding closed embeddings $i$ and $j$. We shall denote by $W^{0}$ and $W$ their underived and derived intersections,

$$
W^{0}=X \times{ }_{S} Y, \quad W=X \times{ }_{S}^{R} Y .
$$

We assume given an Azumaya algebra $\mathscr{A}$ on $S$ which allows us to construct the Azumaya space $\bar{S}=(S, \mathscr{A})$. Let $\bar{X}$ and $\bar{Y}$ be the Azumaya schemes $\left(X,\left.\mathscr{A}\right|_{X}\right)$ and $\left(Y,\left.\mathscr{A}\right|_{Y}\right)$, respectively. They are naturally smooth subvarieties of $\bar{S}$ with corresponding closed embeddings by $\bar{i}$ and $\bar{j}$. The derived intersection $\bar{W}$ of $\bar{X}$ and $\bar{Y}$ inside $\bar{S}$ is given by

$$
\bar{W}=\bar{X} \times \frac{R}{S} \bar{Y}=\left(W,\left.\mathscr{A}\right|_{W}\right) ;
$$

see Example 3.5.

Remark 4.1. Now, assume that the restrictions of $\mathscr{A}$ to $X$ and to $Y$ are split by vector bundles $E_{X}$ and $E_{Y}$ on $X$ and $Y$, respectively:

$$
\left.\mathscr{A}\right|_{X} \cong \operatorname{End}_{X}\left(E_{X}\right),\left.\quad \mathscr{A}\right|_{Y} \cong \operatorname{End}_{Y}\left(E_{Y}\right) .
$$

This data gives rise to isomorphisms of Azumaya schemes $m: X \stackrel{\sim}{\rightarrow} \bar{X}$ and $n: Y \stackrel{\sim}{\rightarrow} \bar{Y}$ which allow us to regard $X$ and $Y$ as smooth subvarieties of either the untwisted space $S=\left(S, \mathscr{O}_{S}\right)$ or the twisted space $\bar{S}=(S, \mathscr{A})$. In the latter case, the closed embedding $i^{\prime}: X \hookrightarrow \bar{S}$ is given by the composite $X \stackrel{m}{\rightarrow} \bar{X} \stackrel{\bar{i}}{\rightarrow} \bar{S}$. A similar composition gives the embedding $j^{\prime}$ of $Y$ into $\bar{S}$.

The given splittings of $\left.\mathscr{A}\right|_{X}$ and $\left.\mathscr{A}\right|_{Y}$ give rise to splittings of $\left.\mathscr{A}\right|_{W}$. These can be regarded as isomorphisms $f$ and $g$ between $W$ and $\bar{W}$. The spaces and morphisms we have discussed so 


\section{Arinkin, A. Căldăraru And M. Hablicsek}

far fit in the following diagram:

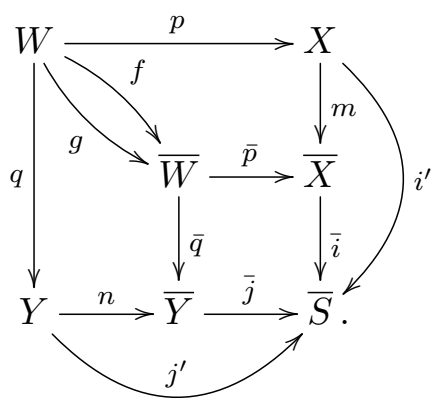

Note that we are not assuming that $\left.\left.E_{X}\right|_{W} \cong E_{Y}\right|_{W}$, hence the morphisms $f$ and $g$ are not necessarily isomorphic. In particular, the entire diagram is not commutative, even though the upper-right and lower-left trapezoids are.

We are interested in comparing the derived intersection $W$ of $X$ and $Y$ inside $S$ with its twisted counterpart, when we consider the intersection as taking place inside $\bar{S}$. Denote by $W^{\prime}$ this latter dg-scheme,

$$
W^{\prime}=X \times \frac{R}{S} Y .
$$

The structure sheaves of $W$ and $W^{\prime}$ (regarded as dg-schemes with morphisms to $X$ and to $Y$ ) are the kernels of $j^{*} i_{*}$ and $j^{\prime *} i_{*}^{\prime}$, respectively, both regarded as functors $\mathbf{D}(X) \rightarrow \mathbf{D}(Y)$. (For a general discussion of the point of view that structure complexes of derived intersections should be understood as kernels of functors, see [ACH14, Section 2].)

Note that we would normally think of $W$ and $W^{\prime}$ as dg-schemes over $X \times Y$ because they are both endowed with morphisms to $X$ and to $Y$. However, since the category of Azumaya spaces does not have absolute products (as opposed to fibered products), it is more natural to think of them as dg-schemes over the pair $(X, Y)$ : such a spaces is endowed with two morphisms, one to $X$ and one to $Y$, and morphisms in this category must make the obvious diagrams commutative.

Remark 4.2. Regarding derived intersections as kernels makes it clear that the dg-scheme $W^{\prime}$ can be taken to be $W$ as an abstract dg-scheme; however, one of the two morphisms $p$ and $q$ has to be modified in order to fix the commutativity of the resulting diagram. Let $\tau$ be the automorphism (in the category of Azumaya dg-schemes) of $W$ given by $\tau=g^{-1} \circ f$. As an abstract Azumaya dg-scheme, $W^{\prime}$ is isomorphic to $W$. However, as a space over $(X, Y)$ it is different from $W$; its projection maps to $X$ and to $Y$ are given by $p$ and $q \circ \tau$, respectively, instead of $p$ and $q$. Note that the resulting diagram commutes:

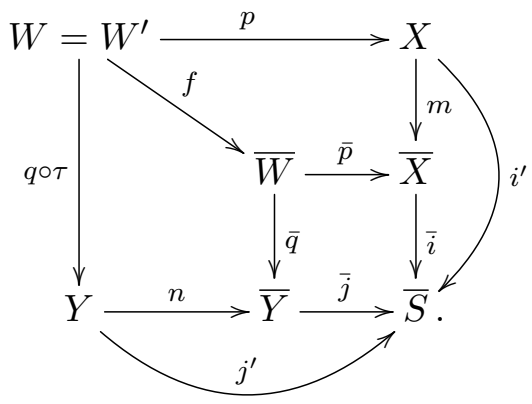

It is easy to understand the automorphism $\tau$ of $W$. At the level of underlying dg-schemes, $\tau$ is the identity, hence $\tau$ must be given by a line bundle $\mathcal{L}$ on $W$; see Example 3.4. Rephrasing the 


\section{Derived INTERSECTIONS AND THE Hodge THEOREM}

commutativity of the above diagram, we get

$$
j^{* *} i_{*}^{\prime}(-) \cong q_{*}\left(p^{*}(-) \otimes \mathcal{L}\right) .
$$

We will call $\mathcal{L}$ the associated line bundle of the twisted derived intersection problem.

Remark 4.3. Line bundles on a dg-scheme $W$ are classified, as in the classical case, by

$$
\operatorname{Pic}(W)=H^{1}\left(W, \mathscr{O}_{W}^{\times}\right)=H^{1}\left(W^{0}, \mathscr{O}_{W}^{\times}\right) .
$$

If $\mathscr{O}_{W}$ is formal and concentrated in non-positive degrees, then the complex $\mathscr{O}_{W}^{\times}$of invertible elements in $\mathscr{O}_{W}$ consists of those whose degree zero part is invertible. (Indeed, $\mathscr{O}_{W}$ is a nilpotent algebra over $\mathscr{O}_{W^{0}}$.) We then have

$$
\operatorname{Pic}(W)=\operatorname{Pic}\left(W^{0}\right) \oplus \bigoplus_{i \geqslant 1} H^{i+1}\left(W^{0}, \mathscr{H}^{-i}\left(\mathscr{O}_{W}\right)\right),
$$

where $\mathscr{H}^{-i}\left(\mathscr{O}_{W}\right)$ is the $-i$ th cohomology sheaf of $\mathscr{O}_{W}$.

In particular, $\operatorname{Pic}\left(W^{0}\right)$ and $H^{2}\left(W^{0}, \mathscr{H}^{-1}\left(\mathscr{O}_{W}\right)\right)$ are direct summands in $\operatorname{Pic}(W)$. It is easy to understand these groups geometrically. If $\mathcal{L}$ is a line bundle on $W$, its zeroth cohomology sheaf $\mathscr{H}^{0}(\mathcal{L})$ is a line bundle on $W^{0}$, and this gives the map $\operatorname{Pic}(W) \rightarrow \operatorname{Pic}\left(W^{0}\right)$. (One can also understand this map as the pull-back via the embedding $W^{0} \hookrightarrow W$.)

Since $\mathscr{O}_{W}$ is formal, there is a projection map $\pi: W \rightarrow W^{0}$. Even though $\pi_{*} \mathscr{O}_{W}$ is a formal complex on $W_{0}$, for a line bundle $\mathcal{L}$ on $W$ the complex $\pi_{*} \mathcal{L}$ need not be formal. The first obstruction to the formality of $\pi_{*} \mathcal{L}$ is its HKR class (see Example 4.5 below), the obstruction to the splitting of $\tau^{\geqslant-1}\left(\pi_{*} \mathcal{L}\right)$, which is a class in

$$
\operatorname{Ext}_{W^{0}}^{2}\left(\mathscr{H}^{0}\left(\pi_{*} \mathcal{L}\right), \mathscr{H}^{-1}\left(\pi_{*} \mathcal{L}\right)\right)=H^{2}\left(W^{0}, \mathscr{H}^{-1}\left(\mathscr{O}_{W}\right)\right) .
$$

In this way, we realize the HKR class for a line bundle $\mathcal{L}$ as a component of the class of $\mathcal{L}$ in $\operatorname{Pic}(W)$.

The main result of this section is the following.

Theorem 4.4. The associated line bundle $\mathcal{L}$ is trivial (or, equivalently, $W \cong W^{\prime}$ as Azumaya dg-schemes over $X \times Y$ ) if conditions (a)-(c) below are satisfied.

(a) $\left.\left.E_{X}\right|_{W^{0}} \cong E_{Y}\right|_{W^{0}}$.

(b) The map $i^{\prime}$ is split to first order.

(c) The natural map $\left.N_{W^{0} / Y} \rightarrow N_{X / S}\right|_{W^{0}}$ of vector bundles on $W^{0}$ is split.

If this is the case, then both $W$ and $W^{\prime}$ are formal dg-schemes over $(X, Y)$ and $W \cong W^{\prime} \cong$ $\operatorname{Tot}_{W^{0}}(\mathbb{E}[-1])$, where

$$
\mathbb{E}=\frac{T_{S}}{T_{X}+T_{Y}}
$$

is the excess intersection bundle for the original intersection problem $X \cap Y$ inside $S$.

Moreover, if $X=Y, i^{\prime}=j^{\prime}$ (that is, $i=j$ and $E_{X}=E_{Y}$ ) and we assume that $i$ is split to first order, then the converse also holds: $W \cong W^{\prime}$ if and only if $i^{\prime}$ is split to first order.

Proof. The proof is essentially the same as the one of the corresponding result in [AC12, ACH14]. Therefore we only review the main points of the proof, highlighting the aspects that are special to the twisted case. 


\section{Arinkin, A. CăLdăRARU And M. Hablicsek}

Let us first review the proof in the untwisted case. There, the proof shows that $W \cong$ $\operatorname{Tot}_{W^{0}}(\mathbb{E}[-1])$ if $i$ is split to first order and condition (c) is satisfied. We shall describe the proof in geometric language. We consider the sequence of maps of dg-spaces over $(X, Y)$

$$
\begin{aligned}
\operatorname{Tot}_{W^{0}}(\mathbb{E}[-1]) \rightarrow & \operatorname{Tot}_{W^{0}}\left(\left.N_{X / S}\right|_{W^{0}}[-1]\right) \cong \operatorname{Tot}_{X}\left(N_{X / S}[-1]\right) \times_{X} W^{0} \\
& \cong\left(X \times_{S} X\right) \times_{X} W^{0} \cong\left(X \times_{S} Y\right) \times_{Y} W^{0} \rightarrow X \times_{S} Y .
\end{aligned}
$$

The first map comes from the splitting of the sequence on $W^{0}$

$$
\left.0 \rightarrow N_{W^{0} / Y} \rightarrow N_{X / S}\right|_{W^{0}} \rightarrow \mathbb{E} \rightarrow 0
$$

while the second isomorphism is the main result of [AC12], and its existence follows from the fact that $i$ is split to first order. (Throughout this proof, we will implicitly assume that all fiber products are derived, dropping the superscript ' $R$ ' for simplicity.)

Having constructed a map $\operatorname{Tot}_{W^{0}}(\mathbb{E}[-1]) \rightarrow X \times{ }_{S} Y$ of dg-spaces over $(X, Y)$, checking that it is a quasi-isomorphism is a local question which can be settled using Koszul resolutions to complete the proof.

Moving over to the twisted case, let $\mathscr{A}$ be an Azumaya algebra over $S$. The same exact proof shows that conditions (b) and (c) imply that $\bar{W} \cong \operatorname{Tot}_{\bar{W}^{0}}(\mathbb{E}[-1])$ as $(\bar{X}, \bar{Y})$ spaces, where placing a bar over an $S$-space $X$ means endowing it with the Azumaya algebra $\left.\mathscr{A}\right|_{X}$. Note that the condition that $\bar{i}$ splits to first order means that the splitting $\pi: X^{(1)} \rightarrow X$ must satisfy the condition that $\left.\pi^{*}\left(\left.\mathscr{A}\right|_{X}\right) \cong \mathscr{A}\right|_{X^{(1)}}$.

The entire discussion above makes no assumptions on the splitting of $\mathscr{A}$ on $X$ or $Y$. Now, assume furthermore that we have fixed splittings $E_{X}$ and $E_{Y}$ of $\left.\mathscr{A}\right|_{X}$ and $\left.\mathscr{A}\right|_{Y}$ as in Remark 4.1. Condition (a) of the theorem means that there is an isomorphism $W^{0} \cong \bar{W}^{0}$ making a commutative diagram of Azumaya spaces

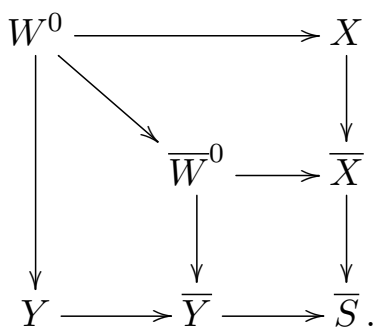

(All the spaces in this diagram are non-derived.) Thus $W^{0}$, as an $(X, Y)$-space, is isomorphic to $\bar{W}^{0}$ as an $(\bar{X}, \bar{Y})$-space. The previous discussion shows that conditions (b) and (c) imply that $\bar{W}$ is the total space of $\mathbb{E}[-1]$ over $\bar{W}^{0}$, as a space over $(\bar{X}, \bar{Y})$. Thus $W^{\prime}$, as the pull-back of $\bar{W}$ over the map of pairs of spaces $(X, Y) \rightarrow(\bar{X}, \bar{Y})$, is isomorphic to $\operatorname{Tot}_{W^{0}}(\mathbb{E}[-1])$ as an $(X, Y)$ space. Since the untwisted map $i$ is split and condition (c) holds, we know that the untwisted intersection $W$ is also isomorphic to $\operatorname{Tot}_{W^{0}}(\mathbb{E}[-1])$. This completes the proof of the fact that

$$
W \cong W^{\prime} \cong \operatorname{Tot}_{W^{0}}(\mathbb{E}[-1]) \text {. }
$$

The reverse implication (the second part of the theorem) follows from the discussion of twisted HKR classes below; see Theorem 4.8.

EXAMPLE 4.5. In the course of the above proof, we used a generalization of Theorem 1.2 which applies for Azumaya spaces. We discuss this generalization below.

Consider the following setup. Let $i: X \hookrightarrow S$ be a closed embedding of smooth schemes over 


\section{Derived INTERSECTIONS AND THE Hodge THEOREM}

a field $\mathbf{k}$ of characteristic either zero or greater than the codimension of $X$ in $S$. Denote the first infinitesimal neighborhood of $X$ in $S$ by $X^{(1)}$.

Let $\mathcal{A}$ be an Azumaya algebra over $S$, providing a map $\bar{i}:\left(X,\left.\mathcal{A}\right|_{X}\right) \hookrightarrow(S, \mathcal{A})$ of Azumaya schemes. Let $E$ be a coherent sheaf on $\left(X,\left.\mathcal{A}\right|_{X}\right)$ that is locally free as an $\mathscr{O}_{X}$-module (a 'twisted vector bundle' for the twisting $\left.\left.\mathcal{A}\right|_{X}\right)$. We are interested in the object $\bar{i}^{*} \bar{i}_{*} E$ of $\mathbf{D}\left(X,\left.\mathcal{A}\right|_{X}\right)$. A local computation with the Koszul complex (similar to the one in [CKS03]) shows that its cohomology sheaves are given by

$$
\mathscr{H}^{-j}\left(\bar{i}^{*} \bar{i}_{*} E\right)=E \otimes \wedge^{j} N^{\vee},
$$

where $N^{\vee}=N_{X / S}^{\vee}$ denotes the conormal bundle of $X$ in $S$. Therefore, we have a triangle in $\mathbf{D}\left(X,\left.\mathcal{A}\right|_{X}\right)$,

$$
E \otimes N^{\vee}[1] \rightarrow \tau^{\geqslant-1} \bar{i}^{*} \bar{i}_{*} E \rightarrow E \rightarrow E \otimes N^{\vee}[2]
$$

We denote the rightmost map in this triangle by

$$
\alpha_{E} \in \operatorname{Ext}_{\left.\mathcal{A}\right|_{X}}^{2}\left(E, E \otimes N^{\vee}\right)
$$

and call it the (twisted) HKR class of $E$, in keeping with the notation of [AC12].

The HKR class satisfies a number of simple properties.

Proposition 4.6. (i) Suppose that $S_{2}$ is another smooth scheme, with a smooth morphism $f: S_{2} \rightarrow S$. Let $X_{2}=f^{-1} X$. Consider the Azumaya algebra $\mathscr{A}_{2}:=f^{*} \mathscr{A}$ on $S_{2}$. Set $E_{2}:=f^{*}(E)$; it is a coherent sheaf on $\left(X_{2},\left.\mathscr{A}_{2}\right|_{X_{2}}\right)$ that is locally free over $\mathscr{O}_{X_{2}}$. Then

$$
\alpha_{E_{2}}=f^{*}\left(\alpha_{E}\right) \in \operatorname{Ext}_{\left.\mathcal{A}_{2}\right|_{X_{2}}}^{2}\left(E_{2}, E_{2} \otimes N_{2}^{\vee}\right),
$$

where $N_{2}=f^{*}(N)$ is the normal bundle to $X_{2} \subset S_{2}$.

(ii) Suppose that $E_{1}$ and $E_{2}$ are two coherent sheaves on $\left(X,\left.\mathscr{A}\right|_{X}\right)$ that are locally free as $\mathscr{O}_{X}$-modules. Then the HKR class of $E_{1} \oplus E_{2}$ is the image of

$$
\left(\alpha_{E_{1}}, \alpha_{E_{2}}\right) \in \operatorname{Ext}_{\left.\mathcal{A}\right|_{X}}^{2}\left(E_{1}, E_{1} \otimes N^{\vee}\right) \oplus \operatorname{Ext}_{\left.\mathcal{A}\right|_{X}}^{2}\left(E_{2}, E_{2} \otimes N^{\vee}\right)
$$

under the natural map to $\operatorname{Ext}^{2}\left(E_{1} \oplus E_{2},\left(E_{1} \oplus E_{2}\right) \otimes N^{\vee}\right)$.

(iii) Suppose that $\mathscr{A}_{1}$ and $\mathscr{A}_{2}$ are two Azumaya algebras on $S$, and let $E_{i}$ be a coherent sheaf on $\left(X,\left.\mathscr{A}_{i}\right|_{X}\right)$ that is locally free as an $\mathscr{O}_{X}$ module $(i=1,2)$. The HKR class of the coherent sheaf $E_{1} \otimes E_{2}$ on $\left(X,\left.\left(\mathscr{A}_{1} \otimes \mathscr{A}_{2}\right)\right|_{X}\right)$ is given by

$$
\alpha_{E_{1} \otimes E_{2}}=\alpha_{E_{1}} \otimes \operatorname{id}_{E_{2}}+\operatorname{id}_{E_{2}} \otimes \alpha_{E_{1}} .
$$

(iv) In the situation of the previous statement, the HKR class of the coherent sheaf $\underline{\operatorname{Hom}}\left(E_{1}, E_{2}\right)$ on $\left(X,\left.\left(\mathscr{A}_{1}^{\mathrm{op}} \otimes \mathscr{A}_{2}\right)\right|_{X}\right)$ is given by

$$
\alpha_{\underline{\text { Hom }}\left(E_{1}, E_{2}\right)}=-\alpha_{E_{1}} \otimes \operatorname{id}_{E_{2}}+\operatorname{id}_{E_{2}} \otimes \alpha_{E_{1}} .
$$

Here $\mathscr{A}_{1}^{\text {op }}$ is the opposite of the Azumaya algebra $\mathscr{A}_{1}$.

While we defined the HKR class using the object $\bar{i}^{*} \bar{i}_{*} E$, we could have used $\bar{i}^{!} \bar{i}_{*} E$ instead. Indeed, a local computation with the Koszul complex shows that

$$
\mathscr{H}^{j}\left(\bar{i}^{*} \bar{i}_{*} E\right)=E \otimes \wedge^{j} N .
$$

Therefore, we have a triangle in $\mathbf{D}\left(X,\left.\mathcal{A}\right|_{X}\right)$

$$
E \rightarrow \tau^{\leqslant 1} \bar{i}^{*} \bar{i}_{*} E \rightarrow E \otimes N[-1] \rightarrow E[1] .
$$




\section{Arinkin, A. CăLdăRARU And M. Hablicsek}

Lemma 4.7. The rightmost map in this triangle is equal to $\alpha_{E}$.

Proof. Consider $E^{\vee}=\underline{\operatorname{Hom}}\left(E, \mathscr{O}_{X}\right)$ as a module over the Azumaya algebra $\left.\mathcal{A}^{\mathrm{op}}\right|_{X}$. If we let $\bar{i}^{\text {op }}$ be the map of Azumaya spaces $\bar{i}^{\mathrm{op}}:\left(X,\left.\mathcal{A}^{\mathrm{op}}\right|_{X}\right) \rightarrow\left(S, \mathcal{A}^{\mathrm{op}}\right)$, we have

$$
\bar{i}^{!} \bar{i}_{*} E=\mathbf{R} \underline{\underline{H o m}}\left(\bar{i}^{\mathrm{op}, *} i_{*}^{\mathrm{op}}\left(E^{\vee}\right), \mathcal{A}^{\mathrm{op}}\right) ;
$$

now, the claim follows from Proposition 4.6(iv).

Finally, we can view the HKR class as the obstruction to extending $E$ to the first infinitesimal neighborhood of the Azumaya scheme $\left(X,\left.\mathscr{A}\right|_{X}\right)$. More precisely, local extensions of $E$ form a gerbe of extensions, which we denote by $\mathcal{E G}$, and the class $[\mathcal{E G}]$ of this gerbe is equal to $\alpha_{E}$. In the 'untwisted' case of ordinary schemes, this gerbe was considered in [AC12]; the case of Azumaya schemes is completely analogous.

Explicitly, we construct the gerbe $\mathcal{E} \mathcal{G}$ on $X$ as follows. Given an open subset $U \subset X$, we denote by $U^{(1)} \subset X^{(1)}$ its first infinitesimal neighborhood. By definition, $\mathcal{E} \mathcal{G}(U)$ is the groupoid whose objects are pairs consisting of an $\left.\mathscr{A}\right|_{U^{(1)}}$-module $E^{(1)}$ that is locally free over $\mathscr{O}_{U^{(1)}}$ and an isomorphism between the restriction $\left.E^{(1)}\right|_{U}$ and the $\left.\mathscr{A}\right|_{U}$-module $\left.E\right|_{U}$. (To simplify the notation, we usually omit this isomorphism.) As $U \subset X$ varies, the groupoids $\mathcal{E} \mathcal{G}(U)$ form a sheaf of groupoids $\mathcal{E} \mathcal{G}$ on $X$. It is locally non-empty (locally, sections exist) and locally connected (any two sections are locally isomorphic). Moreover, the sheaf of automorphisms of any section is naturally identified with

$$
\underline{\operatorname{Hom}}_{\left.\mathcal{A}\right|_{X}}\left(E, E \otimes N^{\vee}\right)=N^{\vee} \text {. }
$$

Thus, $\mathcal{E} \mathcal{G}$ is a gerbe over the sheaf $N^{\vee}$. The class of this gerbe,

$$
[\mathcal{E} \mathcal{G}] \in H^{2}\left(X, N^{\vee}\right)
$$

is equal to $\alpha_{E}$ by an argument that is completely parallel to the proof of [AC12, Proposition 2.6].

Theorem 4.8. In the above setting, assume that the normal bundle $N=N_{X / S}$ extends to the first infinitesimal neighborhood $X^{(1)}$. Then the following statements are equivalent:

(i) The twisted HKR class $\alpha_{E}$ of $E$ vanishes.

(ii) There exists a formality isomorphism

$$
\bar{i}^{*} \bar{i}_{*} E \cong E \otimes \mathbb{S}\left(N^{\vee}[1]\right)
$$

in $\mathbf{D}\left(X,\left.\mathcal{A}\right|_{X}\right)$ (where the action of $\left.\mathcal{A}\right|_{X}$ on the right-hand side comes from its action on $E$ ).

(iii) There exists a formality isomorphism

$$
\bar{i}^{\prime} \bar{i}_{*} E \cong E \otimes \mathbb{S}(N[-1])
$$

in $\mathbf{D}\left(X,\left.\mathcal{A}\right|_{X}\right)$ (where the action of $\left.\mathcal{A}\right|_{X}$ on the right-hand side comes from its action on $E$ ).

(iv) The sheaf $E$ extends to the first infinitesimal neighborhood $X^{(1)}$. Recall that by this we mean that there exists a coherent sheaf $E^{(1)}$ on the Azumaya scheme $\left(X^{(1)},\left.\mathcal{A}\right|_{X^{(1)}}\right)$ that is locally free over $\mathscr{O}_{X^{(1)}}$ and whose restriction to $\left(X,\left.\mathcal{A}\right|_{X}\right)$ is isomorphic to $E$.

Proof. This theorem is entirely similar to the original result [AC12, Theorem 0.7], and we shall leave to the reader the task of filling in the details of the proof.

In the situation of Theorem 4.8, suppose that $E$ splits the Azumaya algebra $\left.\mathcal{A}\right|_{X}$. Recall that this means that $\operatorname{rk}(E)$ is equal to the rank of the Azumaya algebra $\left.\mathcal{A}\right|_{X}$. Note that in this case,

$$
\alpha_{E} \in \operatorname{Ext}_{\left.\mathcal{A}\right|_{X}}^{2}\left(E, E \otimes N^{\vee}\right)=H^{2}\left(X, N^{\vee}\right) .
$$




\section{Derived INTERSECTIONS AND THE Hodge THEOREM}

An extension $E^{(1)}$, if it exists, splits the Azumaya algebra $\left.\mathcal{A}\right|_{X^{(1)}}$. Geometrically, $E$ gives a morphism of Azumaya schemes

$$
\left(X, \mathscr{O}_{X}\right) \rightarrow(S, \mathcal{A})
$$

while $E^{(1)}$ is an extension of this morphism to $\left(X^{(1)}, \mathscr{O}_{X^{(1)}}\right)$. We arrive at the following conclusion. Corollary 4.9. Under the hypotheses of Theorem 4.8, suppose that E splits the Azumaya algebra $\left.\mathcal{A}\right|_{X}$. Then the equivalent conditions of Theorem 4.8 are also equivalent to the following condition:

(v) The morphism

$$
\left(X, \mathscr{O}_{X}\right) \rightarrow(S, \mathcal{A})
$$

given by $E$ fits into the diagram

$$
\left(X, \mathscr{O}_{X}\right) \rightarrow\left(X^{(1)}, \mathscr{O}_{X^{(1)}}\right) \rightarrow(S, \mathcal{A})
$$

(where the left arrow is the closed embedding $X \hookrightarrow X^{(1)}$ ) such that the underlying morphisms of schemes are the closed embeddings

$$
X \hookrightarrow X^{(1)} \hookrightarrow S .
$$

Remark 4.10. In particular, condition (v) implies that the algebra $\left.\mathcal{A}\right|_{X^{(1)}}$ splits. However, the converse is generally not true: it is possible that $\left.\mathcal{A}\right|_{X^{(1)}}$ splits but there is no splitting that restricts to $E$ on $X$.

On the other hand, if every line bundle on $X$ extends to a line bundle on $X^{(1)}$ (for example, if the map $X \rightarrow X^{(1)}$ is split), then condition (v) is equivalent to the weaker condition that $\left.\mathcal{A}\right|_{X^{(1)}}$ splits. Indeed, let $E^{(1)}$ be any splitting module for $\left.\mathcal{A}\right|_{X^{(1)}}$. Its restriction $\left.E^{(1)}\right|_{X}$ is a splitting module for $\left.\mathcal{A}\right|_{X}$; thus there exists a line bundle $L$ on $X$ such that

$$
\left.E \cong E^{(1)}\right|_{X} \otimes L
$$

If $L^{(1)}$ is a line bundle on $X^{(1)}$ that extends $L$, then $E^{(1)} \otimes L^{(1)}$ is a splitting module on $X^{(1)}$ extending $E$.

\section{HKR class in the presence of a section}

Let us return to the situation of Corollary 4.9. Thus $i: X \hookrightarrow S$ is a closed embedding of smooth schemes over a field $\mathbf{k}$ of characteristic either zero or greater than the codimension of $X$ in $S$, $\mathcal{A}$ is an Azumaya algebra on $S$, and $E$ is a splitting module for $\left.\mathcal{A}\right|_{X}$. Above, we have constructed the twisted HKR class of $E$,

$$
\alpha_{E} \in \operatorname{Ext}_{\left.\mathcal{A}\right|_{X}}^{2}\left(E, E \otimes N^{\vee}\right)=H^{2}\left(X, N^{\vee}\right) .
$$

Now, suppose that $E$ admits a nowhere vanishing section $s \in H^{0}(X, E)$. In this case, we can write $\alpha_{E}$ as a product of two classes. Let us sketch this construction.

A section $s$ gives an embedding of vector bundles on $X$,

$$
\mathscr{O}_{X} \rightarrow E, \quad f \mapsto f s .
$$

Denote its cokernel by $E^{\prime}=E /\left(\mathscr{O}_{X} s\right)$. We thus have a short exact sequence

$$
0 \rightarrow \mathscr{O}_{X} \rightarrow E \rightarrow E^{\prime} \rightarrow 0 \text {. }
$$

Let $\beta(E, s) \in \operatorname{Ext}^{1}\left(E^{\prime}, \mathscr{O}_{X}\right)$ be the corresponding cohomology class. Note that $\beta(E, s)$ does not depend on the Azumaya algebra $\mathscr{A}$ or its action on $E$. This is the first of the two classes which decompose $\alpha_{E}$. 


\section{Arinkin, A. CăLdăRARU And M. Hablicsek}

The second cohomology class lies in $\operatorname{Ext}^{1}\left(\left(E^{\prime}\right)^{\vee}, N^{\vee}\right)$. It is the obstruction to the extension of the pair $(E, s)$ to the first infinitesimal neighborhood $X^{(1)}$ of $X$. We can describe it as follows. Since the section $s$ has no zeros, it generates $E$ as an $\left.\mathscr{A}\right|_{X}$-module. The action of $\left.\mathscr{A}\right|_{X}$ on $s$ gives a homomorphism of $\left.\mathscr{A}\right|_{X}$-modules $\left.\mathscr{A}\right|_{X} \rightarrow E$. The kernel of this homomorphism is a left ideal in $\left.\mathscr{A}\right|_{X}$ (the annihilator of $s$ ), which we denote by $\left.\mathscr{I} \subset \mathscr{A}\right|_{X}$.

Consider the problem of deforming $\mathscr{I}$ to an ideal $\left.\mathscr{I}^{(1)} \subset \mathscr{A}\right|_{X^{(1)}}$. Locally in the étale topology, $\mathscr{A}$ splits and such a deformation always exists. Moreover, any two such deformations differ by a section of

$$
\left.\underline{\operatorname{Hom}}_{\mathscr{A}}\right|_{X}\left(\mathscr{I},\left(\left.\mathscr{A}\right|_{X} / \mathscr{I}\right) \otimes N^{\vee}\right) .
$$

Thus, these deformations form a torsor over the sheaf

$$
\underline{\operatorname{Hom}}_{\left.\mathscr{A}\right|_{X}}\left(\mathscr{I},\left(\left.\mathscr{A}\right|_{X} / \mathscr{I}\right) \otimes N^{\vee}\right) \text {. }
$$

Note that the Morita equivalence corresponding to the splitting $E$ identifies this sheaf with the sheaf

$$
\underline{\operatorname{Hom}}_{\mathscr{O}_{X}}\left(\left(E^{\prime}\right)^{\vee}, N^{\vee}\right)
$$

so the class of the torsor is an element

$$
\gamma(E, s) \in \operatorname{Ext}^{1}\left(\left(E^{\prime}\right)^{\vee}, N^{\vee}\right)
$$

as claimed.

Proposition 4.11. The class $\alpha_{E} \in H^{2}\left(X, N^{\vee}\right)=\operatorname{Ext}^{2}\left(N, \mathscr{O}_{X}\right)$ decomposes as the product of $\beta(E, s) \in \operatorname{Ext}^{1}\left(E^{\prime}, \mathscr{O}_{X}\right)$ and $\gamma(E, s) \in \operatorname{Ext}^{1}\left(\left(E^{\prime}\right)^{\vee}, N^{\vee}\right)=\operatorname{Ext}^{1}\left(N, E^{\prime}\right)$.

Proof. The proof is naturally formulated in the language of gerbes. Let us sketch the argument.

Recall that the HKR class $\alpha_{E}$ is equal to the class of the gerbe $\mathcal{E G}$ of extensions of $E$ to the first infinitesimal neighborhood of the Azumaya scheme $\left(X,\left.\mathscr{A}\right|_{X}\right)$. Similarly, we can represent $\gamma(E, s)$ as the class of a gerbe over the complex

$$
C^{\bullet}=\left(C^{0} \rightarrow C^{1}\right):=\left(N^{\vee} \rightarrow E \otimes N^{\vee}\right) .
$$

Recall that a gerbe over a complex of sheaves of abelian groups

$$
C^{\bullet}=\left(C^{0} \rightarrow C^{1}\right)
$$

is a $C^{0}$-gerbe $\mathscr{G}$ and a trivialization of the induced $C^{1}$-gerbe. Such a trivialization can be given by a morphism of sheaves of groupoids $\mathscr{G} \rightarrow \operatorname{Tors}\left(C^{1}\right)$ that is compatible with the 2-action of $C^{0}$. Here, $\operatorname{Tors}\left(C^{1}\right)$ is the trivial $C^{1}$-gerbe; its sections are $C^{1}$-torsors.

In our situation, consider the $N^{\vee}$-gerbe $\mathcal{E G}$. We construct a morphism $\mathcal{E} \mathcal{G} \rightarrow \operatorname{Tors}\left(E \otimes N^{\vee}\right)$ by sending a local section $E^{(1)} \in \mathcal{E} \mathcal{G}(U)$ to the torsor of liftings of $s \in H^{0}\left(X,\left.E\right|_{U}\right)$ to a section $s^{(1)}$ of $E^{(1)}$. In this way, we obtain a gerbe $\mathcal{E G}_{s}$ over the complex $C^{\bullet}$. The corresponding class $\left[\mathcal{E G}_{s}\right]$ belongs to the hypercohomology group $H^{2}\left(X, C^{\bullet}\right)$. Using the quasi-isomorphism $C^{\bullet} \rightarrow E^{\prime} \otimes$ $N^{\vee}[-1]$, we obtain an identification

$$
H^{2}\left(X, C^{\bullet}\right)=H^{1}\left(X, E^{\prime} \otimes N^{\vee}\right),
$$

and it is easy to see that $\left[\mathcal{E G}_{s}\right]$ corresponds to $\gamma(E, s)$ under this isomorphism.

On the other hand, $\mathcal{E} \mathcal{G}$ can be viewed as the gerbe induced by $\mathcal{E G}_{s}$ under the morphism

$$
C^{\bullet} \rightarrow N^{\vee}
$$




\section{DERIVED INTERSECTIONS AND THE HODGE THEOREM}

Accordingly, $[\mathcal{E} \mathcal{G}]$ is the image of $\left[\mathcal{E G}_{s}\right]$ under this morphism. Now, it remains to notice that the composition

$$
E^{\prime} \otimes N^{\vee}[-1] \simeq C^{\bullet} \rightarrow N^{\vee}
$$

is given by product with $\beta(E, s)$.

\section{Applications to Hodge theory}

In this section, we prove our main theorems, Theorems 1.4 and 1.6. We also discuss generalizations of these results to twisted de Rham complexes.

\section{The de Rham complex as a twisted derived intersection}

The following is a restatement of Theorem 1.4.

Theorem 5.1. Let $X$ be a smooth scheme over a perfect field $\mathbf{k}$ of characteristic $p>0$. Let $X^{\prime}$ be its Frobenius twist, and let $\bar{S}=\left(T^{*} X^{\prime}, D\right)$ denote the Azumaya space whose underlying space is the cotangent bundle $T^{*} X^{\prime}$ of $X^{\prime}$. Consider the composite morphism

$$
i^{\prime}: X^{\prime}=\left(X^{\prime}, \mathcal{O}_{X^{\prime}}\right) \stackrel{m}{\longrightarrow}\left(X^{\prime},\left.D\right|_{X^{\prime}}\right) \stackrel{i_{D}}{\longrightarrow} \bar{S}=\left(T^{*} X^{\prime}, D\right) .
$$

Then there are natural isomorphisms in $\mathbf{D}\left(X^{\prime}\right)$,

$$
F_{*} \Omega_{X}^{\cdot} \cong i^{\prime !} i_{*}^{\prime} \mathcal{O}_{X^{\prime}} \cong\left(i^{* *} i_{*}^{\prime} \mathscr{O}_{X^{\prime}}\right)^{\vee} \text {. }
$$

Thus, $F_{*} \Omega_{X}^{\cdot}$ is formal if and only if $i^{\prime *} i_{*}^{\prime} \mathscr{O}_{X^{\prime}}$ is.

Remark 5.2. In geometric language, we can reformulate Theorem 5.1 by saying that the line bundle associated with the twisted derived self-intersection problem of $X^{\prime}$ inside $\bar{S}$ is $\mathcal{L}=\left(F_{*} \Omega_{X}^{*}\right)^{\vee}$. The complex $F_{*} \Omega_{X}^{*}$ is formal if and only if $\mathcal{L}$ is trivial.

We begin with an easy lemma.

Lemma 5.3. Let $f: X \rightarrow Y$ be an affine morphism of schemes, and let $\mathcal{A}$ be a quasi-coherent sheaf of algebras on $X$. For any quasi-coherent sheaf of $\mathcal{A}$-modules $M$, its direct image $f_{*} M$ is a quasi-coherent sheaf of $f_{*} \mathcal{A}$-modules. The functor $f_{*}$ gives an equivalence between the category of quasi-coherent $\mathcal{A}$-modules on $X$ and the category of quasi-coherent $f_{*} \mathscr{A}$-modules on $Y$. It also gives an equivalence between the corresponding derived categories.

Proof of Theorem 1.4. Recall from Remark 2.3 that the sheaf of Azumaya algebras $D$ on $T^{*} X^{\prime}$ satisfies $\phi_{*} D=F_{*} D_{X}$, where $\phi: T^{*} X^{\prime} \rightarrow X^{\prime}$ is the bundle map. We now have

$$
\begin{aligned}
F_{*} \Omega_{X}^{\bullet} & =F_{*} \underline{\operatorname{Rom}}_{\left(X, D_{X}\right)}\left(\mathscr{O}_{X}, \mathscr{O}_{X}\right) \\
& =\mathbf{R} \underline{\operatorname{Hom}}_{\left(X^{\prime}, F_{*} D_{X}\right)}\left(F_{*} \mathcal{O}_{X}, F_{*} \mathcal{O}_{X}\right) \\
& =\mathbf{R} \underline{\operatorname{Hom}}_{\left(X^{\prime}, \phi_{*} D\right)}\left(F_{*} \mathcal{O}_{X}, F_{*} \mathcal{O}_{X}\right), \\
& =\mathbf{R} \underline{\operatorname{Hom}}_{\left(X^{\prime}, \phi_{*} D\right)}\left(\phi_{*} i_{D, *} F_{*} \mathcal{O}_{X}, \phi_{*} i_{D, *} F_{*} \mathcal{O}_{X}\right) \\
& =\phi_{*} \underline{\mathbf{R o m}}_{\left(T^{*} X^{\prime}, D\right)}\left(i_{D, *} F_{*} \mathcal{O}_{X}, i_{D, *} F_{*} \mathcal{O}_{X}\right) .
\end{aligned}
$$

The first equality is from Remark 2.1, the second is Lemma 5.3, the third is the relationship $\phi_{*} D=F_{*} D_{X}$, the fourth is the identity $\phi \circ i_{D}=\mathrm{id}$, and the last is Lemma 5.3 again.

Recall that the functor

$$
i_{D, *}: \quad \mathbf{D}\left(X^{\prime},\left.D\right|_{X^{\prime}}\right) \rightarrow \mathbf{D}\left(T^{*} X^{\prime}, D\right)
$$




\section{Arinkin, A. Căldărara And M. Hablicsek}

admits a right adjoint $i_{D}^{!}$; thus, we have

$$
\mathbf{R} \underline{\operatorname{Hom}}_{\left(T^{*} X^{\prime}, D\right)}\left(i_{D, *} F_{*} \mathscr{O}_{X}, i_{D, *} F_{*} \mathscr{O}_{X}\right)=i_{D, *} \mathbf{R} \underline{\operatorname{Hom}}_{\left(X^{\prime},\left.D\right|_{X^{\prime}}\right)}\left(F_{*} \mathscr{O}_{X}, i_{D}^{!} i_{D, *} F_{*} \mathscr{O}_{X}\right) .
$$

Because $\phi \circ i_{D}=\mathrm{id}$, we conclude that

$$
F_{*} \Omega_{X}^{\cdot}=\mathbf{R} \underline{\operatorname{Hom}}_{\left(X^{\prime},\left.D\right|_{X^{\prime}}\right)}\left(F_{*} \mathcal{O}_{X}, i_{D}^{!} i_{D, *} F_{*} \mathcal{O}_{X}\right) .
$$

At this point, recall that the sheaf of Azumaya algebras $\left.D\right|_{X^{\prime}}$ splits with splitting module $F_{*} \mathcal{O}_{X}$. In fact, $F_{*} \mathscr{O}_{X}=m_{*} \mathscr{O}_{X^{\prime}}$ under the Morita equivalence $\left.\mathscr{O}_{X^{\prime}-\mathfrak{M} \mathfrak{o d}} \stackrel{m_{*}}{\rightarrow} D\right|_{X^{\prime}-\mathfrak{M} \mathfrak{T} \mathfrak{d} \text { of }}$ Remark 2.4, and the inverse equivalence is given by the functor

$$
m^{*}(-)=\underline{\mathbf{R H o m}}_{\left(X^{\prime},\left.D\right|_{X^{\prime}}\right)}\left(F_{*} \mathscr{O}_{X},-\right) \text {. }
$$

Moreover, $m^{*}$ is both a left and a right adjoint to $m_{*}$ because the latter functor is an equivalence. This justifies our writing either $m^{*}$ or $m^{!}$for the functor above.

With these considerations in mind, the result of the above calculation can be rewritten as

$$
\begin{aligned}
F_{*} \Omega_{X}^{\bullet} & =\mathbf{R} \underline{\operatorname{Hom}}_{\left(X^{\prime},\left.D\right|_{X^{\prime}}\right)}\left(F_{*} \mathcal{O}_{X}, i_{D}^{!} i_{D, *} F_{*} \mathcal{O}_{X}\right)=m^{!} i_{D^{\prime}}^{i_{D, *}} m_{*} \mathcal{O}_{X^{\prime}} \\
& =i^{\prime !} i_{*}^{\prime} \mathscr{O}_{X^{\prime}} .
\end{aligned}
$$

The statement that $i^{\prime \prime} i_{*}^{\prime} \mathscr{O}_{X^{\prime}} \cong\left(i^{\prime *} i_{*}^{\prime} \mathscr{O}_{X^{\prime}}\right)^{\vee}$ is a standard form of duality. Finally, the discussion in Remark 4.2 implies that the associated line bundle of this twisted derived intersection is $\left(F_{*} \Omega_{X}^{\bullet}\right)^{\vee}$.

Remark 5.4. The algebra $i^{i} i_{*} \mathscr{O}_{X^{\prime}}$ is always formal, that is,

$$
i^{!} i_{*} \mathscr{O}_{X^{\prime}} \cong \mathbb{S}_{X^{\prime}}\left(\Omega_{X^{\prime}}^{1}[-1]\right)
$$

Since $i^{\prime} i_{*}^{\prime} \mathscr{O}_{X^{\prime}}$ and $i^{!} i_{*} \mathscr{O}_{X^{\prime}}$ have the same cohomology sheaves anyway, the formality of $F_{*} \Omega_{X}^{\bullet}$ is equivalent to the existence of an isomorphism

$$
i^{\prime !} i_{*}^{\prime} \mathscr{O}_{X^{\prime}} \cong i^{!} i_{*} \mathscr{O}_{X^{\prime}}
$$

or, dually, the existence of an isomorphism

$$
i^{\prime *} i_{*}^{\prime} \mathscr{O}_{X^{\prime}} \cong i^{*} i_{*} \mathscr{O}_{X^{\prime}}
$$

This justifies our statement that the above formality statements are equivalent to the triviality of $\mathcal{L}$.

\section{Splitting on the first infinitesimal neighborhood}

In this section, we investigate under what circumstances the complex $F_{*} \Omega_{X}^{\cdot}=i^{\prime} i_{*}^{\prime} \mathscr{O}_{X^{\prime}}$ is formal. We prove the following theorem, which is essentially Theorem 1.6.

ThEOREM 5.5. Let $X$ be a smooth variety over a perfect field $\mathbf{k}$ of characteristic $p>\operatorname{dim} X$. Then the following statements are equivalent:

(i) The variety $X$ lifts to $W_{2}(\mathbf{k})$.

(ii) We have $\alpha=0$, where $\alpha \in H^{2}\left(X^{\prime}, T_{X^{\prime}}\right)$ is the class of the extension

$$
0 \rightarrow \mathcal{O}_{X^{\prime}} \rightarrow F_{*} \mathcal{O}_{X} \stackrel{F_{*} d}{\longrightarrow} F_{*} Z^{1} \rightarrow \Omega_{X^{\prime}}^{1} \rightarrow 0 .
$$

Here $Z^{1}$ denotes the image of $d$ in $\Omega_{X}^{1}$.

(iii) The map $i^{\prime}$ splits to first order.

(iv) The algebra $D$ splits on the first infinitesimal neighborhood of $X^{\prime}$ in $T^{*} X^{\prime}$. 


\section{Derived INTERSECTIONS AND THE Hodge THEOREM}

(v) The line bundle $\mathcal{L}$ associated with the twisted derived self-intersection problem of $X^{\prime}$ inside $\bar{S}$ is trivial.

(vi) The complex $F_{*} \Omega_{X}^{*}=i^{\prime !} i_{*}^{\prime} \mathcal{O}_{X^{\prime}}$ is formal in $\mathbf{D}\left(X^{\prime}\right)$; that is, there exists an isomorphism

$$
F_{*} \Omega_{X}^{\cdot}=i^{\prime !} i_{*}^{\prime} \mathcal{O}_{X^{\prime}} \cong \mathbb{S}\left(\Omega_{X^{\prime}}^{1}[-1]\right)=i^{!} i_{*} \mathcal{O}_{X^{\prime}}
$$

Proof. We apply Theorem 4.4 to the problem of studying the formality of the twisted derived selfintersection of $X^{\prime}$ inside $\bar{S}=\left(T^{*} X^{\prime}, D\right)$. Conditions (a) and (c) of Theorem 4.4 are automatically satisfied. Condition (b) is statement (iii)above, and the theorem thus shows that statement (iii) is equivalent to statement (vi).

Remark 4.10 shows that statement (iii) is also equivalent to statement (iv).

The equivalence of statements (v) and (vi) follows from Remark 5.2.

Note that $F_{*} \Omega_{X}^{*} \cong i^{\prime !} i_{*}^{\prime} \mathscr{O}_{X^{\prime}}$, so we have

$$
\tau^{\leqslant 1}\left(F_{*} \Omega_{X}^{\cdot}\right) \cong \tau^{\leqslant 1}\left(i^{\prime !} i_{*}^{\prime} \mathscr{O}_{X^{\prime}}\right) .
$$

The class of the extension in statement (ii) is the obstruction to splitting the former; the twisted HKR class $\alpha_{\mathscr{O}_{X^{\prime}}}$ is by definition the obstruction to splitting the latter. We conclude that statement (ii) is equivalent to $\alpha_{\mathscr{O}_{X^{\prime}}}=0$. Moreover, Theorem 4.8 shows that $\alpha_{\mathscr{O}_{X^{\prime}}}=0$ is equivalent to statement (iv). Thus, we have proved that statements (ii) and (iv) are equivalent.

Finally, the equivalence of statements (i) and (ii) is proved in the original paper of DeligneIllusie [DI87].

\section{The twisted de Rham complex}

We have seen in the previous section that the (dual of the) Frobenius push-forward of the de Rham complex can be directly regarded as a line bundle over the derived self-intersection of the zero section in the twisted cotangent bundle $\left(T^{*} X^{\prime}, D\right)$. In this section, we argue that the twisted de Rham complex of Barannikov-Kontsevich can also be regarded in a similar way as a line bundle over a derived intersection in $\left(T^{*} X^{\prime}, D\right)$, as in Theorem 1.9. This allows us to give a geometric realization of the Hodge-de Rham spectral sequence for categories of singularities.

We start with a scheme $X$ which is smooth over a perfect field $\mathbf{k}$ of characteristic $p>0$. We do not assume anymore that $X$ is proper over $k$. We choose a regular function $f: X \rightarrow \mathbb{A}^{1}$ on $X$, sometimes called the superpotential in physics literature.

From these data, we construct the context for a twisted derived intersection as in Section 4. As the ambient space $S$, we take the total space of the cotangent bundle $T^{*} X^{\prime}$ of the Frobenius twist $X^{\prime}$. The Azumaya algebra on $S$ is $\mathscr{A}=D$, the ring of differential operators on $X$. As one of the two subvarieties of $S$, we take the zero section $X^{\prime} \hookrightarrow T^{*} X^{\prime}$.

Let $f^{\prime}=\pi^{*} f$, where $\pi: X^{\prime} \rightarrow X$ is as in Section 2. We take as the second subvariety of $T^{*} X^{\prime}$ the graph $X_{f}^{\prime}$ of the exact 1 -form $d f^{\prime}$. As an abstract variety, $X_{f}^{\prime}$ is isomorphic to $X^{\prime}$, with a different embedding into $T^{*} X^{\prime}$. As above, we denote the embeddings $X^{\prime} \hookrightarrow T^{*} X^{\prime}$ and $X_{f}^{\prime} \hookrightarrow T^{*} X^{\prime}$ by $i$ and $j$, respectively. Notice that the underived intersection (which was previously denoted by $W^{0}$ ) is the critical locus Crit $f^{\prime}$ of $f^{\prime}$.

Locally on $X^{\prime}$, any vector field $\partial$ gives rise to a function on $T^{*} X^{\prime}$, linear in the fibers of the map $T^{*} X^{\prime} \rightarrow X^{\prime}$. The zero section of $T^{*} X^{\prime}$ is locally cut out by the equations $\partial^{\prime}=0$ for $\partial \in T_{X^{\prime}}$. Similarly, the graph of $d f^{\prime}$ is cut out by the equations $\partial^{\prime}-\partial^{\prime}\left(f^{\prime}\right)=0$.

Denote by $\psi$ the automorphism of the ring of differential operators $D_{X}$ on $X$ that acts on functions $\mathscr{O}_{X} \subset D_{X}$ as the identity and on vector fields as $\partial \mapsto \partial-\partial f$. Its action on the center 


\section{Arinkin, A. Căldăraru And M. Hablicsek}

$\mathscr{O}_{T^{*} X^{\prime}}$ of $D$ is made explicit by the following lemma.

Lemma 5.6. The automorphism $s$ of $\mathscr{O}_{T^{*} X^{\prime}}$ induced by $\psi$ acts on functions as the identity and on vector fields $\partial^{\prime} \in T_{X^{\prime}}$ as $\partial^{\prime} \mapsto \partial^{\prime}-\partial^{\prime}\left(f^{\prime}\right)$.

Proof. As discussed in Remark 2.2, the map $\pi$ induces an isomorphism between the sheaves of vector fields on $X$ and on $X^{\prime}$. For a local vector field $\partial^{\prime}$ on $X^{\prime}$, we write $\partial$ for the corresponding local vector field on $X$. A straightforward calculation shows that

$$
\begin{aligned}
\psi\left(g^{p}\right) & =g^{p}, \\
\psi\left(\partial^{p}\right) & =\partial^{p}-\partial^{[p]} f-(\partial f)^{p}
\end{aligned}
$$

for any $g \in \mathscr{O}_{X}$ and $\partial \in T_{X}$. Therefore,

$$
\psi\left(\partial^{p}-\partial^{[p]}\right)=\partial^{p}-\partial^{[p]} f-(\partial f)^{p}-\left(\partial^{[p]}-\partial^{[p]} f\right)=\partial^{p}-\partial^{[p]}-(\partial f)^{p},
$$

where $\partial^{p}-\partial^{[p]}$ and $\partial^{p}-\partial^{[p]}-(\partial f)^{p}$ are exactly the images of $\partial^{\prime}$ and $\partial^{\prime}-\partial^{\prime}\left(f^{\prime}\right)$ under the identification $\bar{\iota}: \mathscr{O}_{T^{*} X^{\prime}} \rightarrow Z\left(D_{X}\right)$.

Remark 5.7. We shall write $L$ for the $D_{X}$-module $\psi_{*} \mathscr{O}_{X}$. As an $\mathscr{O}_{X}$-module, it is isomorphic to $\mathscr{O}_{X}$; however, the action of $\partial \in T_{X}$ is modified in such a way that $\partial$ acts on a local section $g$ of $\mathscr{O}_{X}$ by

$$
\partial . g=\partial(g)-\partial f .
$$

We shall think of $\psi$ as an automorphism of the twisted space $\left(T^{*} X^{\prime}, D\right)$, acting by $s$ on the underlying space $T^{*} X^{\prime}$ and by $\psi$ on $D$. By Lemma 5.6, we have $s\left(X^{\prime}\right)=X_{f}^{\prime}$. Since $F_{*} \mathscr{O}_{X}$ splits the restriction $i^{*} D$ of $D$, we see that $F_{*} L=F_{*} \psi_{*} \mathscr{O}_{X}$ splits $j^{*} D$.

We conclude that we are in the context of Section 4: we have an ambient space $\bar{S}=\left(T^{*} X^{\prime}, D\right)$ with two subvarieties $X^{\prime}$ and $X_{f}^{\prime}$ such that $D$ splits on both of them. We will use the notation of Section 4 from now on. In particular, we shall denote by $i^{\prime}$ and $j^{\prime}$ the twisted embeddings of $X^{\prime}$ and $X_{f}^{\prime}$, respectively, into $\bar{S}$ and by $W$ and $W^{\prime}$ the untwisted and twisted derived intersections of $X^{\prime}$ and $X_{f}^{\prime}$ inside $S$ and $\bar{S}$, respectively.

In the remainder of this section, we show that the structure complex of $W$ is the dual of $\Omega_{X^{\prime}, \wedge d f^{\prime}}^{\circ}$ and that the associated line bundle $\mathcal{L}$ for the above intersection problem is the dual of $F_{*} \Omega_{X, d-\wedge d f}^{\bullet}$.

To explain the above relationship, we need to remind the reader about the two complexes that appear in Theorem 1.9. The first is the de Rham complex of the $D_{X}$-module $L$ given by the complex of sheaves on $X$ :

$$
\mathrm{d} R(L)=\Omega_{X, d-\wedge d f}^{\cdot}=0 \longrightarrow \Omega_{X}^{0} \stackrel{d-\wedge d f}{\longrightarrow} \Omega_{X}^{1} \stackrel{d-\wedge d f}{\longrightarrow} \Omega_{X}^{2} \longrightarrow \cdots ;
$$

we view it as a twisted analogue of the de Rham complex of $X$. The second is the analogue of the formal complex $\mathbb{S}\left(\Omega_{X^{\prime}}^{1}[-1]\right)$; it is the complex of coherent sheaves on $X^{\prime}$ given by

$$
\Omega_{X^{\prime}, \wedge d f^{\prime}}^{\cdot}=0 \longrightarrow \Omega_{X^{\prime}}^{0} \stackrel{\wedge d f^{\prime}}{\longrightarrow} \Omega_{X^{\prime}}^{1} \stackrel{\wedge d f^{\prime}}{\longrightarrow} \Omega_{X^{\prime}}^{2} \longrightarrow \cdots .
$$

The following theorem rephrases Theorem 1.9.

THEOREm 5.8. Let $W$ and $W^{\prime}$ be the derived intersections of the zero section and the graph of $d f^{\prime}$ inside $\left(T^{*} X^{\prime}, \mathscr{O}\right)$ and $\left(T^{*} X^{\prime}, D\right)$, respectively. Then

(i) the structure complex $\mathscr{O}_{W}$ of $W$ is the dual (over $X^{\prime}$ ) of $\Omega_{X^{\prime}, \wedge d f^{\prime}}$, and 


\section{Derived INTERSECTIONS AND THE Hodge THEOREM}

(ii) the associated line bundle $\mathcal{L}$ of the twisted derived intersection problem is the dual (again, over $X^{\prime}$ ) of $F_{*} \Omega_{X, d-\wedge d f}^{*}$.

If, furthermore, $\mathcal{L}$ is trivial, then the two complexes $\Omega_{X^{\prime}, \wedge d f^{\prime}}$ and $F_{*} \Omega_{X, d-\wedge d f}^{\bullet}$ are quasi-isomorphic.

Proof. Part (i) is an easy calculation with an explicit Koszul resolution of $j_{*} \mathscr{O}_{X^{\prime}}$, so we will concentrate on part (ii). We have the following sequence of equalities:

$$
\begin{aligned}
F_{*} \Omega_{X, d-\wedge d f}^{\bullet} & =F_{*} \underline{\operatorname{Rom}}_{\left(X, D_{X}\right)}\left(\mathcal{O}_{X}, L\right) \\
& =\mathbf{R} \underline{\operatorname{Hom}}_{\left(X^{\prime}, F_{*} D_{X}\right)}\left(F_{*} \mathcal{O}_{X}, F_{*} L\right) \\
& =\mathbf{R} \underline{\operatorname{Hom}}_{\left(X^{\prime}, \phi_{*} D\right)}\left(F_{*} \mathcal{O}_{X}, F_{*} L\right),
\end{aligned}
$$

where the first equality follows from Remark 2.1, the second is Lemma 5.3, and the third is $F_{*} D_{X}=\phi_{*} D$.

Following the notation of Section 3, we denote by $i_{D}$ and $j_{D}$ the embeddings of the twisted spaces $\left(X^{\prime}, i^{*} D\right)$ and $\left(X^{\prime}, j^{*} D\right)$, respectively, into $\left(T^{*} X^{\prime}, D\right)$. Note that $\phi \circ j_{D}=\mathrm{id}=\phi \circ i_{D}$ because both $i$ and $j$ are sections of the bundle map $\phi: T^{*} X^{\prime} \rightarrow X^{\prime}$. We have

$$
\begin{aligned}
F_{*} \Omega_{X, d-\wedge d f}^{\cdot} & =\underline{\mathbf{R}}_{\underline{\operatorname{Hom}}_{\left(X^{\prime}, \phi_{*} D\right)}}\left(F_{*} \mathcal{O}_{X}, F_{*} L\right) \\
& =\mathbf{R} \underline{\operatorname{Hom}}_{\left(X^{\prime}, \phi_{*} D\right)}\left(\phi_{*} i_{D, *} F_{*} \mathcal{O}_{X}, \phi_{*} j_{D, *} F_{*} L\right) \\
& =\phi_{*} \mathbf{R} \underline{\operatorname{Hom}}_{\left(T^{*} X^{\prime}, D\right)}\left(i_{D, *} F_{*} \mathcal{O}_{X}, j_{D, *} F_{*} L\right) \\
& =\phi_{*} i_{*} \mathbf{R} \underline{\operatorname{Hom}}_{\left(X^{\prime}, i^{*} D\right)}\left(F_{*} \mathcal{O}_{X}, i_{D}^{!} j_{D, *} F_{*} L\right) \\
& =\mathbf{R} \underline{\operatorname{Hom}}_{\left(X^{\prime}, i^{*} D\right)}\left(F_{*} \mathcal{O}_{X}, i_{D}^{!} j_{D, *} F_{*} L\right),
\end{aligned}
$$

where the second equality comes from $\phi \circ j=\mathrm{id}=\phi \circ i$, the third is Lemma 5.3, the fourth is adjunction, and the fifth is again $\phi \circ i=\mathrm{id}$. Since $F_{*} \mathscr{O}_{X}$ and $F_{*} L$ are splitting modules of $i^{*} D$ and $j^{*} D$, respectively, a calculation similar to one in the proof of Theorem 5.1 shows that

$$
F_{*} \Omega_{X, d-\wedge d f}^{\cdot}=m ! i_{D}^{!} j_{D, *} n_{*} \mathcal{O}_{X^{\prime}}=i^{\prime !} j_{*}^{\prime} \mathcal{O}_{X^{\prime}} .
$$

This implies that the dual of $F_{*} \Omega_{X, d-\wedge d f}^{\circ}$ is the associated line bundle of the twisted derived intersection. (We have denoted by $n$ the isomorphism $\left(X^{\prime}, \mathscr{O}_{X^{\prime}}\right) \cong\left(X^{\prime}, j^{*} D\right)$ induced by the splitting module $F_{*} L$ of $j^{*} D$.)

Recall that in the case of the untwisted de Rham complex, the main result was a complete characterization of when the associated line bundle of the intersection is trivial. Specifically, we argued that $F_{*} \Omega_{X}^{*}$ and $\mathbb{S}\left(\Omega_{X^{\prime}}^{1}[-1]\right)$ are isomorphic if and only if $X$ lifts to $W_{2}(\mathbf{k})$.

In the case of the twisted de Rham complex, the natural question is whether there exists a quasi-isomorphism

$$
F_{*} \Omega_{X, d-\wedge d f}^{\bullet} \cong \Omega_{X^{\prime}, \wedge d f^{\prime}} .
$$

Unfortunately, it seems hard to give a precise criterion for the existence of such an isomorphism.

Note, however, that Ogus and Vologodsky prove (see the proof of [OV07, Theorem 4.23]) that if we start with a variety $X$ and a proper map $f: X \rightarrow \mathbb{A}^{1}$ defined over a field of characteristic zero and consider reductions of this pair $(X, f)$ to characteristic $p$, then the above quasi-isomorphism exists for all $p$ that are large enough. They then use this result to derive the BarannikovKontsevich theorem.

In this paper, we consider a slightly different question: we ask for conditions when both complexes $F_{*} \Omega_{X, d-\wedge d f}^{\cdot}$ and $\Omega_{X^{\prime}, \wedge d f^{\prime}}$ are formal. This turns out to be a special case of the above 


\section{Arinkin, A. Căldăraru And M. Hablicsek}

problem: these two complexes have isomorphic cohomology sheaves anyway; therefore, once they are known to be formal, they are automatically quasi-isomorphic.

As a particular case, the untwisted de Rham theorem corresponds to $f=0$. Indeed, if $f=0$, the complex

$$
\Omega_{X^{\prime}, \wedge d f^{\prime}}^{\bullet} \simeq \mathbb{S}\left(\Omega_{X^{\prime}}^{1}[-1]\right)
$$

is clearly formal and its cohomology sheaves are isomorphic to the cohomology sheaves as $F_{*} \Omega_{X}^{*}$. Therefore, if $f=0$, the two complexes are quasi-isomorphic if and only if they are both formal.

The theorem below is our main result concerning the formality of twisted de Rham complexes.

TheOREM 5.9. Let $X$ be a smooth variety over a perfect field of characteristic $p$, and let $f: X \rightarrow \mathbb{A}^{1}$ be a morphism. Assume that the following conditions hold:

(a) $p>\operatorname{dim}(X)$.

(b) Both $X$ and $f$ lift to $W_{2}(\mathbf{k})$.

(c) The critical locus $Z=$ Crit $f$ is scheme-theoretically smooth.

(d) The morphism $f$ is constant on each connected component of $Z$.

(e) The embedding $Z \hookrightarrow X$ is split to first order; in other words, the following short exact sequence splits:

$$
\left.0 \rightarrow T_{Z} \rightarrow T_{X}\right|_{Z} \rightarrow N_{Z / X} \rightarrow 0 .
$$

Then the complexes $F_{*} \Omega_{X, d-\wedge d f}^{\cdot}$ and $\Omega_{X^{\prime}, \wedge d f^{\prime}}$ are both formal and quasi-isomorphic.

Remark 5.10. If conditions (a) and (c) in the statement of the theorem are satisfied, the formality of $\Omega_{X^{\prime}, \wedge d f^{\prime}}^{\circ}$ implies condition (e) by [ACH14, Theorem 1.8], thus providing a partial converse of Theorem 5.9.

Corollary 5.11. Suppose that $X$ and $f$ satisfy the conditions (a)-(e) of Theorem 5.9. Then there are isomorphisms

$$
\mathbf{R}^{i} \Gamma\left(X, \Omega_{X, d-\wedge d f}^{\cdot}\right) \cong \mathbf{R}^{i} \Gamma\left(X, \Omega_{X^{\prime}, \wedge d f^{\prime}}^{\bullet} \cong \bigoplus_{i-c=p+q} H^{p}\left(Z^{\prime}, \Omega_{Z^{\prime}}^{q} \otimes \omega\right)\right.
$$

where $c$ denotes the codimension of $Z$ in $X$ and $\omega$ denotes the relative dualizing complex of the embedding $Z^{\prime} \hookrightarrow X^{\prime}$.

Proof. Indeed, it is easy to see that since $Z^{\prime}$ is smooth, the cohomology sheaves of the complex $\Omega_{X^{\prime}, \wedge d f^{\prime}}$ are given by

$$
H^{i}\left(\Omega_{X^{\prime}, \wedge d f^{\prime}}^{\bullet}\right)=\Omega_{Z^{\prime}}^{i-c} \otimes \omega .
$$

Corollary 5.11 can now be transferred to characteristic zero, giving Theorem 1.10. For the reader's convenience we repeat the statement of Theorem 1.10 below.

Theorem 5.12 (Theorem 1.10). Let $X$ be a smooth variety over a field $\mathbf{k}$ of characteristic zero, and let $f: X \rightarrow \mathbb{A}^{1}$ be a proper morphism. Suppose that the following conditions are satisfied:

(a) The critical locus $Z=$ Crit $f$ is scheme-theoretically smooth.

(b) The embedding $Z \hookrightarrow X$ is split to first order; that is, the short exact sequence below splits:

$$
\left.0 \rightarrow T_{Z} \rightarrow T_{X}\right|_{Z} \rightarrow N_{Z / X} \rightarrow 0 \text {. }
$$




\section{DERIVED INTERSECTIONS AND THE Hodge THEOREM}

Then there exist isomorphisms

$$
\mathbf{R}^{i} \Gamma\left(X, \Omega_{X, d-\wedge d f}^{\bullet}\right) \cong \mathbf{R}^{i} \Gamma\left(X, \Omega_{X, \wedge d f}^{\bullet}\right) \cong \bigoplus_{i-c=p+q} H^{p}\left(Z, \Omega_{Z}^{q} \otimes \omega\right)
$$

where $c$ denotes the codimension of $Z$ in $X$ and $\omega$ denotes the relative dualizing complex of the embedding $Z \hookrightarrow X$.

Proof. The theorem is deduced from the characteristic $p$ statement (Corollary 5.11) in a manner parallel to the argument of [OV07]. Note that this argument relies on a very subtle statement concerning specialization of the de Rham cohomology to characteristic $p$; see the first claim of [OV07, Theorem 4.23].

Remark 5.13. The isomorphism

$$
\mathbf{R}^{i} \Gamma\left(X, \Omega_{X, \wedge d f}^{\bullet}\right) \cong \bigoplus_{i-c=p+q} H^{p}\left(Z, \Omega_{Z}^{q} \otimes \omega\right)
$$

follows directly from [ACH14, Theorem 1.8] (which is the untwisted version of Theorem 4.4). Thus, Theorem 1.10 is also implied by the combination of [ACH14, Theorem 1.8] and the Barannikov-Kontsevich theorem (Theorem 1.8).

Let us now prove Theorem 5.9. Its proof is a straightforward application of Theorem 4.4 (which in turn generalizes the main result of [ACH14], similar in nature also to the main result of [Gri14]). The only non-trivial aspect of the proof is checking condition (a) of Theorem 4.4; that is, we need to verify that the splitting modules of the Azumaya algebra $D$ along $X^{\prime}$ and $X_{f}^{\prime}$ agree along the (non-derived) intersection $Z^{\prime}=X^{\prime} \cap X_{f}^{\prime}$. This requires a study of exponential functions in positive characteristic.

Let $J \subset \mathscr{O}_{X}$ be the Jacobian ideal of $f$, that is, the ideal of the closed subscheme $Z \subset X$. Put

$$
J^{[p]}=\left\langle g^{p} \mid g \in J\right\rangle,
$$

so that $J^{[p]}$ is the ideal of the closed subscheme $Z^{[p]}=F^{-1}\left(Z^{\prime}\right) \subset X$. Note that $J^{[p]} \subset \mathscr{O}_{X}$ is a sub- $D_{X}$-module.

Recall that $D$ is trivialized along the zero section $X^{\prime}$ of $T^{*} X^{\prime}$ by the module $E=F_{*} \mathscr{O}_{X}$. By Lemma 5.6, the action of $\psi$ on $D$ moves the zero section $X^{\prime}$ to the section $X_{f}^{\prime}$, and hence the module $E_{f}=F_{*} \psi_{*} \mathscr{O}_{X}=F_{*} L$ is a splitting module of $D$ along $X_{f}^{\prime}$. To complete the proof of Theorem 5.9, we need to show that $\left.\left.E\right|_{Z^{\prime}} \cong E_{f}\right|_{Z^{\prime}}$ as $\left.F_{*} D\right|_{Z^{\prime}}$-modules. Since the map $F: X \rightarrow X^{\prime}$ is affine, this is equivalent to finding an isomorphism of $D_{X}$-modules

$$
\mathscr{O}_{X} / J^{[p]} \simeq L / L \cdot J^{[p]} .
$$

Recall that $L$ is a trivial rank one $\mathscr{O}_{X}$-module, but derivations $\partial \in T_{X} \subset D_{X}$ act on it by $\partial-\partial f$. Thus, an isomorphism

$$
\mathscr{O}_{X} / J^{[p]} \simeq L / L \cdot J^{[p]}
$$

is given by multiplication by an invertible function

$$
g \in\left(\mathscr{O}_{X} / J^{[p]}\right)^{\times}
$$

that must satisfy the differential equation

$$
d \log (g)=d f \bmod J^{[p]}
$$

as usual, $d \log (g)=d g / g$. 


\section{Arinkin, A. CăLdăRARU And M. Hablicsek}

We will call a solution $g$ to the above differential equation an approximate exponential of $f$. Observe that in characteristic $p$ the usual power series for $\exp (f)$ is not defined past the $p$ th term.

At this point, the existence of an approximate exponential follows from the work of Ogus and Vologodsky [OV07, Proposition 4.28]. This completes the proof of Theorem 5.9. Since the relevant part of [OV07] is spread over several claims, we restate their result and sketch its proof below for the reader's convenience.

Proposition 5.14. As before, let $X$ be a smooth variety over a perfect field $\mathbf{k}$ of characteristic $p$, and let $f: X \rightarrow \mathbb{A}^{1}$ be a morphism. Denote by $Z=$ Crit $f$ the critical locus of $f$, and let $J \subset \mathscr{O}_{X}$ be the Jacobian ideal of $f$. Suppose that the following conditions hold:

(a) Both $X$ and $f$ lift to $W_{2}(\mathbf{k})$.

(b) The morphism $f$ is constant on each reduced connected component of $Z$.

(c) We have $p>c n$, where $c$ is the maximal codimension of $Z$ in $X$ and $n$ is the smallest number such that $(\sqrt{J})^{n} \subset J$.

Under these assumptions, an approximate exponential exists: there is a $g \in\left(\mathscr{O}_{X} / J^{[p]}\right)^{\times}$such that

$$
d \log (g)=d f \bmod J^{[p]}
$$

Proof. Without loss of generality, we may assume that $Z$ is connected. Subtracting a constant from $f$, we may assume $f \in \sqrt{J}$.

Define $\alpha \in \mathscr{O}_{X} / J^{[p]}$ as follows. Locally on $X$, there exists a lift $\tilde{F}$ of the Frobenius map $F$ to $W_{2}(\mathbf{k}) ;$ put

$$
\alpha=\frac{1}{p}\left(\tilde{F}(\tilde{f})-\tilde{f}^{p}\right) \bmod p,
$$

where $\tilde{f}$ is the lift of $f$. One can check that $\alpha$ does not depend on the choice of $\tilde{F}$, and therefore the local expressions glue to define $\alpha$ globally.

Now, define $g$ by the formula

$$
g=\operatorname{hexp}(f) \cdot\left(1+\alpha+\frac{\alpha^{2}}{2 !}+\cdots+\frac{\alpha^{p-1}}{(p-1) !}\right) .
$$

Here

$$
h \exp (x)=\exp \left(x+\frac{x^{p}}{p}+\frac{x^{p^{2}}}{p^{2}}+\cdots\right)
$$

is the Artin-Hasse exponential [AH28]. It is a formal power series whose coefficients are rational numbers whose denominators are not divisible by $p$, and therefore hexp $(x)$ makes sense over fields of characteristic $p$.

It is not hard to check that $g$ is indeed an approximate exponential. Indeed,

$$
d \log (\operatorname{hexp}(f))=\left(1+f^{p-1}+f^{p^{2}-1}+\cdots\right) d f=\left(1+f^{p-1}\right) d f \bmod J^{[p]}
$$

because $f^{p^{2}-1} \in J^{[p]}$. Local calculation shows that $\alpha^{c} \in(\sqrt{J})^{[p]}$; therefore $\alpha^{p-1}=0 \bmod J^{[p]}$, which implies that

$$
d \log \left(1+\alpha+\frac{\alpha^{2}}{2 !}+\cdots+\frac{\alpha^{p-1}}{(p-1) !}\right)=d \alpha \bmod J^{[p]}
$$




\section{DERIVED INTERSECTIONS AND THE HODGE THEOREM}

It remains to notice that $d \alpha=f^{p-1} d f$, and hence

$$
\begin{aligned}
d \log (g) & =d \log (\operatorname{hexp}(f))+d \log \left(1+\alpha+\frac{\alpha^{2}}{2 !}+\cdots+\frac{\alpha^{p-1}}{(p-1) !}\right) \\
& =d f \bmod J^{[p]},
\end{aligned}
$$

as claimed.

Remark 5.15. We conclude the paper with an observation concerning approximate exponentials. As we have already mentioned, $\alpha^{p-1}=0 \bmod J^{[p]}$ under the hypotheses of Proposition 5.14. For this reason, we can rewrite the formula for $g$ as

$$
g=h \exp (f) \cdot \operatorname{hexp}(\alpha) .
$$

This suggests a different informal approach to finding an approximate exponential as an infinite product. Namely, suppose that there exist lifts of $\tilde{X}$ and $\tilde{f}$ not just to the ring $W_{2}(\mathbf{k})$ of second Witt vectors, but to the ring $W(\mathbf{k})$ of infinite-level Witt vectors. We can now consider lifts $\tilde{F}$ of the Frobenius map $F$ to $W(\mathbf{k})$; generally speaking, such $\tilde{F}$ exists only locally.

Consider the sequence

$$
\begin{aligned}
h_{0} & =\tilde{f}, \\
h_{1} & =\frac{1}{p}\left(\tilde{F}(\tilde{f})-h_{0}^{p}\right), \\
h_{2} & =\frac{1}{p^{2}}\left(\tilde{F}^{2}(\tilde{f})-h_{0}^{p^{2}}-p \cdot h_{1}^{p}\right), \\
& \vdots \\
h_{n} & =\frac{1}{p^{n}}\left(\tilde{F}^{n}(\tilde{f})-\sum_{j=0}^{n-1} p^{j} h_{j}^{p^{n-j}}\right),
\end{aligned}
$$

These expressions are well defined over $W(\mathbf{k})$ (in other words, the expressions in parentheses are divisible by the corresponding power of $p$ ). One may consider as a candidate for an approximate exponential of $f$ the reduction modulo $p$ of the infinite product

$$
\tilde{g}=\operatorname{hexp}\left(h_{0}\right) \cdot \operatorname{hexp}\left(h_{1}\right) \cdot h \exp \left(h_{2}\right) \cdots .
$$

(Here we are completely ignoring convergence issues.) The approximate exponential used in Proposition 5.14 is the reduction of the truncated product $\operatorname{hexp}\left(h_{0}\right) \cdot \operatorname{hexp}\left(h_{1}\right)$.

The recursive expressions for $h_{i}$ imply the identity

$$
\tilde{g}=\exp \left(\tilde{f}+\frac{\tilde{F}(\tilde{f})}{p}+\frac{\tilde{F}^{2}(\tilde{f})}{p^{2}}+\cdots\right)
$$

over $W(\mathbf{k})\left[p^{-1}\right]$. Taking the derivative of both sides, we see that

$$
d \tilde{g}=d \tilde{f}+\frac{d \tilde{F}(\tilde{f})}{p}+\frac{d \tilde{F}^{2}(\tilde{f})}{p^{2}}+\cdots
$$

It is easy to see that $d \tilde{F}^{k}(\tilde{f})$ is actually divisible by $p^{k}$ over $W(\mathbf{k})$, so the right-hand side of the formula makes sense over $W(\mathbf{k})$. Moreover, the reduction of $p^{-k} d \tilde{F}^{k}(\tilde{f})$ modulo $p$ vanishes modulo $J^{[p]}$. This justifies the expectation that $\tilde{g}$ is an approximate exponential of $\tilde{f}$. 


\section{Arinkin, A. CăLd ĂRARU AND M. Hablicsek}

The above formulas are perhaps more conceptual than the truncated expression used in Proposition 5.14. Unfortunately, there are two issues with this approach, and it is not clear how to make it rigorous. First, the above infinite products and sums may diverge. Second, even when the expression for $\tilde{g}$ is well defined, it may depend on the choice of local lift $\tilde{F}$. In some cases, these issues can be resolved by restricting the class of lifts $\tilde{F}$ that one considers.

\section{ACKNOWLEDGEMENTS}

The entire project described in this paper was sparked by a comment and question by Mircea Mustață, for which we are grateful. The second author has benefited from a stimulating conversation with Michael Groechenig. The idea of Remark 5.15 arose from conversations with Taylor Dupuy.

\section{REFERENCES}

AC12 D. Arinkin and A. Căldăraru, When is the self-intersection of a subvariety a fibration?, Adv. Math. 231 (2012), no. 2, 815-842; https://doi.org/10.1016/j.aim.2012.05.014.

ACH14 D. Arinkin, A. Căldăraru, and M. Hablicsek, Formality of derived intersections and the orbifold HKR isomorphism, 2014, arXiv:1412.5233.

AH28 E. Artin and H. Hasse, Die beiden Ergänzungssätze zum Reziprozitätsgesetz der $l^{n}$-ten Potenzreste im Körper der $l^{n}$-ten Einheitswurzeln, Abh. Math. Sem. Univ. Hamburg 6 (1928), no. 1, 146-162; https://doi.org/10.1007/BF02940607.

BK98 S. Barannikov and M. Kontsevich, Frobenius manifolds and formality of Lie algebras of polyvector fields, Int. Math. Res. Not. 1998 (1998), no. 4, 201-215; https://doi.org/10.1155/ S1073792898000166.

BMR08 R. Bezrukavnikov, I. Mirković, and D. Rumynin, Localization of modules for a semisimple Lie algebra in prime characteristic, Ann. of Math. 167 (2008), no. 3, 945-991; https://doi.org/ 10.4007/annals.2008.167.945.

Căl00 A. Căldăraru, Derived categories of twisted sheaves on Calabi-Yau manifolds, Ph.D. Thesis, Cornell University, 2000, available at http://www.math.wisc.edu/ andreic/publications/ ThesisSingleSpaced.pdf.

CKS03 A. Căldăraru, S. Katz, and E. Sharpe, D-branes, B fields, and Ext groups, Adv. Theor. Math. Phys. 7 (2003), no. 3, 381-404; https://doi.org/10.4310/ATMP.2003.v7.n3.a1.

DI87 P. Deligne and L. Illusie, Relèvements modulo $p^{2}$ et décomposition du complexe de de Rham, Invent. Math. 89 (1987), no. 2, 247-270; https://doi.org/10.1007/BF01389078.

Gri14 J. Grivaux, Formality of derived intersections, Doc. Math. 19 (2014), 1003-1016; https://www. math.uni-bielefeld.de/documenta/vol-19/33.html.

OV07 A. Ogus and V. Vologodsky, Nonabelian Hodge theory in characteristic p, Publ. Math. Inst. Hautes Études Sci. (2007), no. 106, 1-138; https://doi.org/10.1007/s10240-007-0010-z.

Sab99 C. Sabbah, On a twisted de Rham complex, Tohoku Math. J. 51 (1999), no. 1, 125-140; https: //doi.org/10.2748/tmj/1178224856. 


\section{DERIVED INTERSECTIONS AND THE HODGE THEOREM}

Dima Arinkin arinkin@math.wisc.edu

Mathematics Department, University of Wisconsin-Madison, 480 Lincoln Drive, Madison, WI 53706, USA

Andrei Căldăraru andreic@math.wisc.edu

Mathematics Department, University of Wisconsin-Madison, 480 Lincoln Drive, Madison, WI 53706, USA

Márton Hablicsek mhabli@math.upenn.edu

Mathematics Department, University of Pennsylvania, David Rittenhouse Lab, 209 S. 33rd Street, Philadelphia, PA 19104, USA 